\title{
WHAT DRIVES THE EXPANSION OF GIANT H II REGIONS?: A STUDY OF STELLAR FEEDBACK IN 30 DORADUS
}

\author{
Laura A. Lopez ${ }^{1}$, Mark R. Krumholz ${ }^{1}$, Alberto D. Bolatto ${ }^{2}$, J. Xavier Prochaska ${ }^{1,3}$, and Enrico Ramirez-Ruiz ${ }^{1}$ \\ ${ }^{1}$ Department of Astronomy and Astrophysics, University of California Santa Cruz, 159 Interdisciplinary Sciences Building, 1156 High Street, Santa Cruz, \\ CA95064, USA; lopez@astro.ucsc.edu \\ 2 Department of Astronomy, University of Maryland, College Park, MD 20742, USA \\ ${ }^{3}$ UCO/Lick Observatory, University of California, 1156 High Street, Santa Cruz, CA 95064, USA \\ Received 2010 August 12; accepted 2011 February 19; published 2011 March 28
}

\begin{abstract}
Observations show that star formation is an inefficient and slow process. This result can be attributed to the injection of energy and momentum by stars that prevents free-fall collapse of molecular clouds. The mechanism of this stellar feedback is debated theoretically; possible sources of pressure include the classical warm H II gas, the hot gas generated by shock heating from stellar winds and supernovae, direct radiation of stars, and the dust-processed radiation field trapped inside the $\mathrm{H}$ II shell. In this paper, we measure observationally the pressures associated with each component listed above across the giant $\mathrm{H}$ II region 30 Doradus in the Large Magellanic Cloud. We exploit high-resolution, multi-wavelength images (radio, infrared, optical, ultraviolet, and X-ray) to map these pressures as a function of position. We find that radiation pressure dominates within $75 \mathrm{pc}$ of the central star cluster, R136, while the H II gas pressure dominates at larger radii. By contrast, the dust-processed radiation pressure and hot gas pressure are generally weak and not dynamically important, although the hot gas pressure may have played a more significant role at early times. Based on the low X-ray gas pressures, we demonstrate that the hot gas is only partially confined and must be leaking out the $\mathrm{H}$ II shell. Additionally, we consider the implications of a dominant radiation pressure on the early dynamics of 30 Doradus.
\end{abstract}

Key words: galaxies: star clusters: general - H II regions - ISM: individual objects (30 Doradus) - ISM: kinematics and dynamics - stars: formation - stars: massive

Online-only material: color figures

\section{INTRODUCTION}

Molecular clouds contain the coolest and densest gas in the universe, and thus they are the sites where stars form. The physical properties of these clouds set the initial conditions for protostellar collapse and may define the stellar initial mass function (IMF; Motte et al. 1998; Testi \& Sargent 1998; Onishi et al. 2002). The massive stars formed there eventually end in supernova ( $\mathrm{SN}$ ) explosions, injecting mechanical energy and chemically enriching the interstellar medium (ISM). Therefore, molecular clouds shape the entire stellar life cycle, and an understanding of their properties and dynamics is key to probe galactic evolution.

Observational evidence shows that star formation is an inefficient and slow process. Only 5\%-10\% of available molecular cloud mass is converted into stars over the cloud lifetime ${ }^{4}$ (Williams \& McKee 1997), and only $\sim 2 \%$ of the gas is converted to stars in one free-fall time across several orders of magnitude in density (Zuckerman \& Evans 1974; Krumholz \& Tan 2007). This inefficiency can be attributed to the internal processes of $\mathrm{H}$ II regions that disrupt their host molecular clouds (e.g., Matzner 2002; Krumholz et al. 2006), but the mode of this stellar feedback remains uncertain.

Broadly, there are several possible sources of internal energy and momentum that may drive the dynamics of $\mathrm{H}$ II regions: the direct radiation from stars (e.g., Jijina \& Adams

\footnotetext{
4 Cloud lifetime is debated contentiously in the literature. Observational estimates range from a single free-fall time (Elmegreen 2000; Hartmann et al. 2001; Ballesteros-Paredes \& Hartmann 2007) to several free-fall times (Tan et al. 2006). However, there is a consensus that only a few percent of gas is converted in either timescale.
}

1996; Krumholz \& Matzner 2009), the dust-processed infrared radiation trapped inside an $\mathrm{H}_{\mathrm{II}}$ shell (Thompson et al. 2005; Murray et al. 2010a; Andrews \& Thompson 2011), the warm gas ionized by massive stars (e.g., Whitworth 1979; Dale et al. 2005), the hot gas shock-heated by stellar winds and SNe (e.g., Yorke et al. 1989; Harper-Clark \& Murray 2009), and protostellar outflows/jets (e.g., Quillen et al. 2005; Cunningham et al. 2006; Li \& Nakamura 2006; Nakamura \& Li 2008; Wang et al. 2010). Each of these mechanisms has been considered individually in the literature, but no observational analyses have ever compared the relative contribution of all these components within $\mathrm{H}$ II regions.

In this paper, we investigate the role of the stellar feedback mechanisms listed above in the giant $\mathrm{H}$ II region 30 Doradus in the nearby Large Magellanic Cloud (LMC). Several properties of the LMC make it a favorable target: the LMC's proximity $(\sim 50 \mathrm{kpc})$ ensures that individual point sources can be resolved while maintaining the capability of mapping the diffuse emission at sub-pc scales. Additionally, the LMC has a face-on orientation and a low column density (a few $\left.\times 10^{21} \mathrm{~cm}^{-2}\right)$ that limits line-of-sight confusion. Given these advantages, the LMC (and thus 30 Doradus) has been surveyed at many wavelengths at high spatial resolution, and we can exploit these data to compare observationally all the feedback mechanisms and how they vary with position across 30 Doradus.

The paper is organized as follows: Section 1.1 gives relevant background on the source, 30 Doradus, and describes why this source is a good "test case" for our analyses. In Section 2, we present the multi-wavelength data utilized in our work to assess the dynamical role of all the possible stellar feedback mechanisms. Section 3 outlines how we utilize these images to 


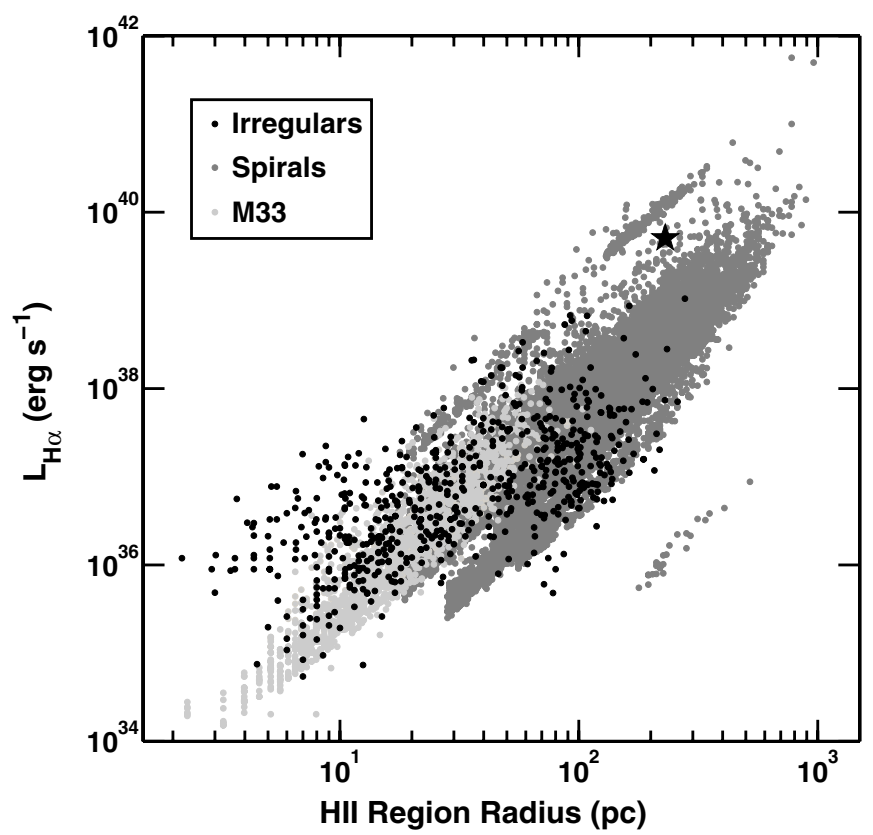

Figure 1. $\mathrm{H} \alpha$ luminosity vs. $\mathrm{H}$ II region radius for $\sim 22,000 \mathrm{H}$ II regions in 70 nearby (distances $\lesssim 30 \mathrm{Mpc}$ ) galaxies. The black star at the top right denotes 30 Doradus. It is the brightest $\mathrm{H}$ II region in the irregular galaxies by nearly an order of magnitude, and it is more luminous than $\approx 99 \%$ of the $\mathrm{H}$ II regions in the spiral galaxies. We note that none of the data are corrected for reddening. The plotted data were compiled from the following references. LMC and SMC: Kennicutt \& Hodge (1986); Sextans A: Hodge et al. (1994); NGC 6822: Hodge et al. (1989a); Holmberg II: Hodge et al. (1994); GR8: Hodge et al. (1989b); DDO 47, Leo A, Sextans B, DDO 167, DDO 168, DDO 187: Strobel et al. (1991); DDO 53 Strobel et al. (1990); 56 spirals: Knapen et al. (2003), Bradley et al. (2006); M33: Wyder et al. (1997), Hodge et al. (1999). We utilized the distances from Kennicutt et al. (2008) to convert from $\mathrm{H} \alpha$ flux to luminosity.

calculate the pressures associated with each feedback component across 30 Doradus. Section 4 gives the results from our analyses, and Section 5 discusses the implications of our findings, including evidence of X-ray gas leakage from the $\mathrm{H}$ II region (Section 5.1) and the role of radiation pressure in $\mathrm{H}$ II region dynamics (Section 5.3). Additionally, we articulate the different ways one can define radiation pressure, and how these definitions can lead to divergent results in Section 5.2. Finally, we summarize and conclude our analysis in Section 6.

\subsection{Background on 30 Doradus}

30 Doradus is the most massive and largest $\mathrm{H}$ II region in the Local Group. The primary star cluster powering 30 Doradus is NGC 2070, with 2400 OB stars (Parker 1993), an ionizing photon luminosity of $S=4.5 \times 10^{51}$ photons $\mathrm{s}^{-1}$ (Walborn 1991) and a bolometric luminosity of $7.8 \times 10^{7} L_{\odot}$ (Malumuth \& Heap 1994). The IMF of NGC 2070 has masses up to $120 M_{\odot}$ (Massey \& Hunter 1998), and the stellar population may be the result of several epochs of star formation (Walborn \& Blades 1997). At the core of NGC 2070 is R136, the densest concentration of very massive stars known, with a central density of $5.5 \times 10^{4} M_{\odot} \mathrm{pc}^{-3}$ (Hunter et al. 1995); R136 hosts at least 39 O3-type stars and at least $10 \mathrm{WR}$ stars in its core $(\sim 2.5 \mathrm{pc}$ in diameter; Massey \& Hunter 1998).

To provide context for how 30 Doradus compares to other local $\mathrm{H}$ II regions, Figure 1 plots $\mathrm{H} \alpha$ luminosity versus $\mathrm{H}$ II region radius for $\sim 22,000 \mathrm{H}$ II regions in 70 nearby (distances $\lesssim 30 \mathrm{Mpc}$ ) galaxies (see references in the figure caption). Morphologically, this galaxy sample is comprised of 13 irregulars/ dwarf irregulars and 57 spirals. The black star near the top right denotes 30 Doradus. It is the brightest in $\mathrm{H} \alpha$ of the $613 \mathrm{H}$ II regions in the irregulars by nearly an order of magnitude. Relative to the $\mathrm{H}$ II regions in spirals (including M33), 30 Doradus has a greater $\mathrm{H} \alpha$ luminosity than $\sim 99 \%$ of that sample.

The nebula that surrounds the central star cluster has a complex morphology across the electromagnetic spectrum. Figure 2 shows a three-color image of 30 Doradus, with the Spitzer Space Telescope $8 \mu \mathrm{m}$ IRAC band in red, $\mathrm{H} \alpha$ in green, and soft X-rays (0.5-2.0 keV) in blue (details of these data are given in Section 2). Large- and small-scale structures are evident from thin ionized gas and dust filaments of arcsecond widths to cavities a few arcminutes across filled with hot X-ray gas. The warm ionized gas has several shell-like structures, and many of these are expanding with high velocities $\left(\sim 100-300 \mathrm{~km} \mathrm{~s}^{-1}\right.$; Chu \& Kennicutt 1994), suggesting that past SN explosions have occurred in the region. In addition to a large ionized gas mass $\left(\sim 8 \times 10^{5} M_{\odot}\right.$; Kennicutt 1984$)$, the 30 Doradus nebula also has $\sim 10^{6} M_{\odot}$ of CO (Johansson et al. 1998). The CO(1-0) maps of 30 Doradus have revealed 33 molecular cloud complexes in the $\mathrm{H}$ II region, and in particular, two elongated clouds of CO mass $\sim 4 \times 10^{5} M_{\odot}$ that form a "ridge" west and north of R136 (see the $\mathrm{CO}$ contours in Figure 2). Estimates of the radius $R_{\mathrm{H} \text { II }}$ of the nebula range from $\sim 110 \mathrm{pc}$ (Brandl 2005; using a revised value of $D=50 \mathrm{kpc}$ ) to $\sim 185 \mathrm{pc}$ (Kennicutt 1984). The nearly factor of two uncertainty in $R_{\mathrm{H} \text { II }}$ arises from the complex shape that precludes accurate determination of the radius. In this paper, we assume $\mathrm{H}$ II $=150 \mathrm{pc}$.

The properties of 30 Doradus described above demonstrate why this HiI region is an ideal candidate for assessing the feedback mechanisms of massive stars. The shear number and energetic output of the OB stars facilitate a detailed study of the effects of radiation, winds, SNe, ionization fronts, etc. Additionally, the proximity of 30 Doradus enables a resolved view of the processes and dynamics associated with starburst activity that was common in the early universe (e.g., Meurer et al. 1997; Shapley et al. 2003). Indeed, the relatively instantaneous formation of the concentrated massive stars in R136 makes 30 Doradus a "mini-starburst" (Leitherer 1997).

\section{DATA}

We analyzed images of 30 Doradus at several wavelengths. A brief description of these data is given below.

\subsection{Optical}

We compiled optical photometric data on 30 Doradus from three separate observational programs. For the central $35^{\prime \prime} \times$ $35^{\prime \prime}$ around R136 (with right ascension $\alpha=05^{\mathrm{h}} 38^{\mathrm{m}} 45^{\mathrm{s}} .5$ and declination $-69^{\circ} 06^{\prime} 02^{\prime \prime}$. 7 ), we utilize the photometric results of Malumuth \& Heap (1994) from Hubble Space Telescope (HST) Planetary Camera (PC) observations. These authors identified over 800 stars within this area and obtained a bolometric luminosity $L_{\text {bol }}=7.8 \times 10^{7} L_{\odot}$ for their sources.

At larger distances from R136 out to a few arcminutes, we employ the $U B V$ photometric data of Parker (1993). The optical images of 30 Doradus from Parker (1993) were obtained at the $0.9 \mathrm{~m}$ telescope at Cerro Tololo Inter-American Observatory (CTIO), with a field of view of 2'.6 $\times 4^{\prime} .1$ and 0.49 pixel $^{-1}$. We followed the analyses of Parker \& Garmany (1993) to convert their measured apparent $U B V$ magnitudes to absolute bolometric magnitudes. 


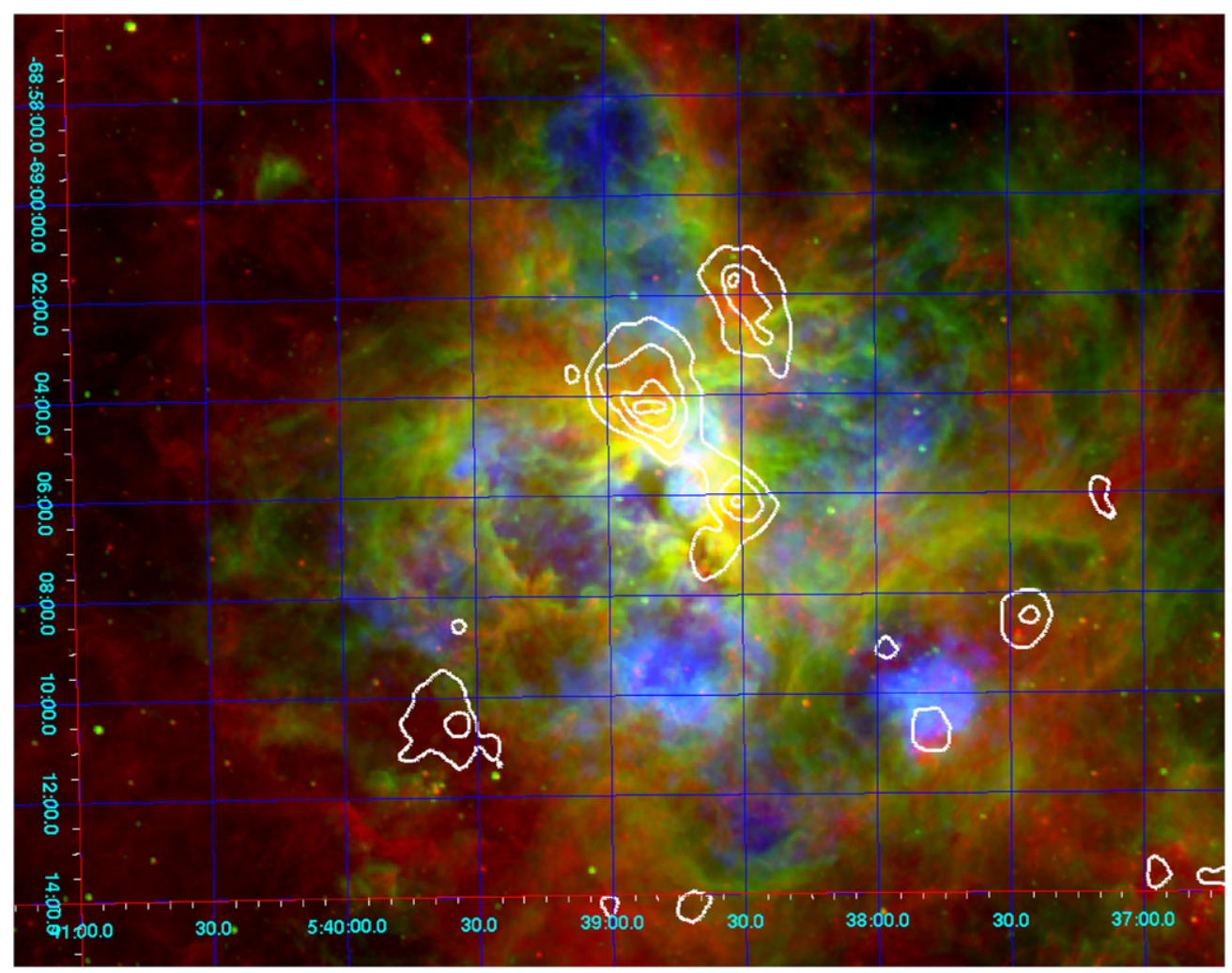

Figure 2. Three-color image of 30 Doradus: MIPS $8 \mu \mathrm{m}$ (red), $\mathrm{H} \alpha$ (green), and $0.5-8 \mathrm{keV} \mathrm{X-rays} \mathrm{(blue).} \mathrm{White} \mathrm{contours} \mathrm{show} \mathrm{the}{ }^{12} \mathrm{CO}(1-0)$ emission (Johansson et al. 1998) in the region. Both large- and small-scale structures are evident. north is up, east is left.

For the area outside the field of Parker (1993), we use the $U B V$ data of Selman \& Melnick (2005). These observations were taken with the Wide Field Imager on the MPG/ESO $2.2 \mathrm{~m}$ telescope at La Silla, out to half a degree away from R136 with $0^{\prime \prime} .238$ pixel $^{-1}$. Thus, the three data sets combined provide a full coverage of 30 Doradus in the $U, B$, and $V$ bands.

To illustrate the $\mathrm{H}$ II region structure, we show the $\mathrm{H} \alpha$ emission of 30 Doradus in Figure 2. This narrowband image (at $6563 \AA$, with $30 \AA$ full-width at half-maximum) was taken with the University of Michigan/CTIO $61 \mathrm{~cm}$ Curtis Schmidt Telescope at CTIO as part of the Magellanic Cloud Emission Line Survey (Smith \& MCELS Team 1998). The total integration time was $600 \mathrm{~s}$, and the reduced image has a resolution of $2^{\prime \prime}$ pixel $^{-1}$.

\subsection{Infrared}

Infrared images of 30 Doradus were obtained through the Spitzer Space Telescope Legacy project Surveying the Agents of Galaxy Evolution (Meixner et al. 2006) of the LMC. The survey covered an area of $\sim 7 \times 7$ degrees of the LMC with the Infrared Array Camera (IRAC; Fazio et al. 2004) and the Multiband Imaging Photometer (MIPS; Rieke et al. 2004). Images were taken in all bands of IRAC (3.6, 4.5, 5.8, and $7.9 \mu \mathrm{m})$ and of MIPS $(24,70$, and $160 \mu \mathrm{m})$ at two epochs in 2005. For our analyses, we used the combined mosaics of both epochs with $1^{\prime \prime} .2$ pixel $^{-1}$ in the 3.6 and $7.9 \mu \mathrm{m}$ IRAC images and 2".49 pixel $^{-1}$ and 4 ." 8 pixel $^{-1}$ in the MIPS $24 \mu \mathrm{m}$ and $70 \mu \mathrm{m}$ images, respectively.

\subsection{Radio}

30 Doradus was observed with the Australian Telescope Compact Array (ATCA) as part of a $4.8 \mathrm{GHz}$ and $8.64 \mathrm{GHz}$ survey of the LMC (Dickel et al. 2005). This program used two array configurations that provided 19 antenna spacings, and these ATCA observations were combined with the Parkes $64 \mathrm{~m}$ telescope data of Haynes et al. (1991) to account for extended structure missed by the interferometric observations. For our analyses, we utilized the resulting ATCA+Parkes $8.64 \mathrm{GHz}(3.5 \mathrm{~cm}) \mathrm{im}$ age of 30 Doradus, which had a Gaussian beam of FWHM 22" and an average rms noise level of $0.5 \mathrm{mJy} \mathrm{beam}^{-1}$. We note that higher resolution ATCA observations of 30 Doradus have been taken by Lazendic et al. (2003), but we have opted to use the ATCA+Parkes image of Dickel et al. (2005) as the latter is more sensitive to the low surface-brightness outskirts of 30 Doradus.

\subsection{X-ray}

30 Doradus was observed using the Chandra Advanced CCD Imaging Spectrometer (ACIS) in 2006 January for $\approx 94 \mathrm{ks}$ total (ObsIDs 5906 [13 ks], 7263 [43 ks], and 7264 [38 ks]; PI: L. Townsley) in the timed-exposure VFaint mode. The spatial resolution of the Chandra ACIS images is 0.'492 pixel $^{-1}$. Data reduction and analysis was performed using the Chandra Interactive Analysis of Observations (CIAO) Version 4.1. We followed the CIAO data preparation thread to reprocess the Level $2 \mathrm{X}$-ray data and merge the three observations together. Figure 3 shows the resulting soft X-ray band $(0.5-2.0 \mathrm{keV})$ image following these analyses. Seventy-four point sources were identified in the reprocessed images using the CIAO command wavdetect (a source detection algorithm using wavelet analysis; Freeman et al. 2002); we excluded the identified point sources in our spectral analyses.

To produce a global X-ray spectrum of 30 Doradus, we extracted Chandra spectra using the CIAO command specextract. 


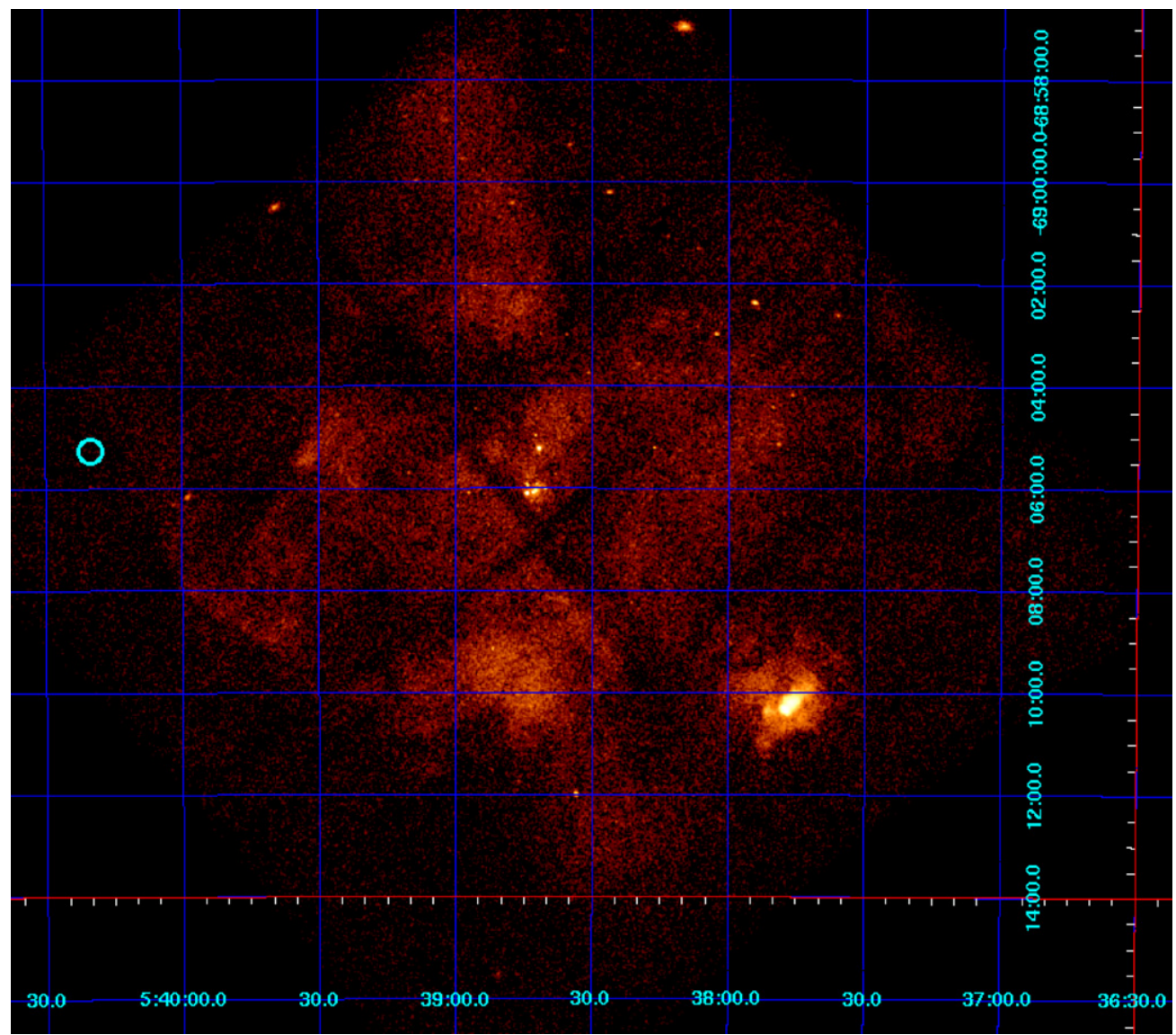

Figure 3. Chandra ACIS-I X-ray soft band $(0.5-2.0 \mathrm{keV})$ image of 30 Doradus. The image was binned by a factor of four so that structures are visually apparent. The cyan circle $\approx 2^{\prime}$ east of 30 Doradus is the area where background spectra were extracted. north is up, east is left.

Background spectra were also produced from a circular region of radius $\approx 15^{\prime \prime}$ that is $\approx 2^{\prime}$ east of 30 Doradus, and these were subtracted from the source spectra. Additionally, we removed the counts of the 74 point sources identified above. The resulting spectra were modeled simultaneously as an absorbed, variable-abundance plasma in collisional ionization equilibrium (XSPEC model components phabs and vmekal) in XSPEC Version 12.4.0. Figure 4 gives the spectra with the best-fit model (with $\chi^{2}=619$ with 396 degrees of freedom (dof)) overplotted. We found a best-fit absorbing column density of $N_{\mathrm{H}}=1.5_{-0.2}^{+0.3} \times 10^{21} \mathrm{~cm}^{-2}$ and an X-ray gas temperature of $k T_{\mathrm{X}}=0.64_{-0.02}^{+0.03} \mathrm{keV}$. The absorption-corrected soft-band $(0.5-2.0 \mathrm{keV})$ luminosity of the diffuse emission is $L_{\mathrm{X}}=4.5 \times 10^{36} \mathrm{erg} \mathrm{s}^{-1}$.

Previous Chandra X-ray analysis of 30 Doradus was reported by Townsley et al. (2006a, 2006b) for a different set of observations (ObsIDs 22 and 62520) totalling $~ 24 \mathrm{ks}$. By fitting the X-ray spectra of many diffuse regions across 30 Doradus, they found best-fit absorbing columns of $N_{\mathrm{H}}=1-6 \times 10^{21} \mathrm{~cm}^{-2}$, temperatures of $k T_{\mathrm{X}} \sim 0.3-0.9 \mathrm{keV}$, and absorption-corrected luminosities $(0.5-2.0 \mathrm{keV})$ of $\log L_{\mathrm{X}}=34.2-37.0 \mathrm{erg} \mathrm{s}^{-1}$. Thus, our values are fairly consistent with those of Townsley et al.

\section{METHODOLOGY}

To assess how feedback varies spatially across 30 Doradus, we separate the source into 441 regions (see Figure 5). The area of the individual regions was selected to ensure sufficient signal to noise across the analyzed wavebands; we chose the width of

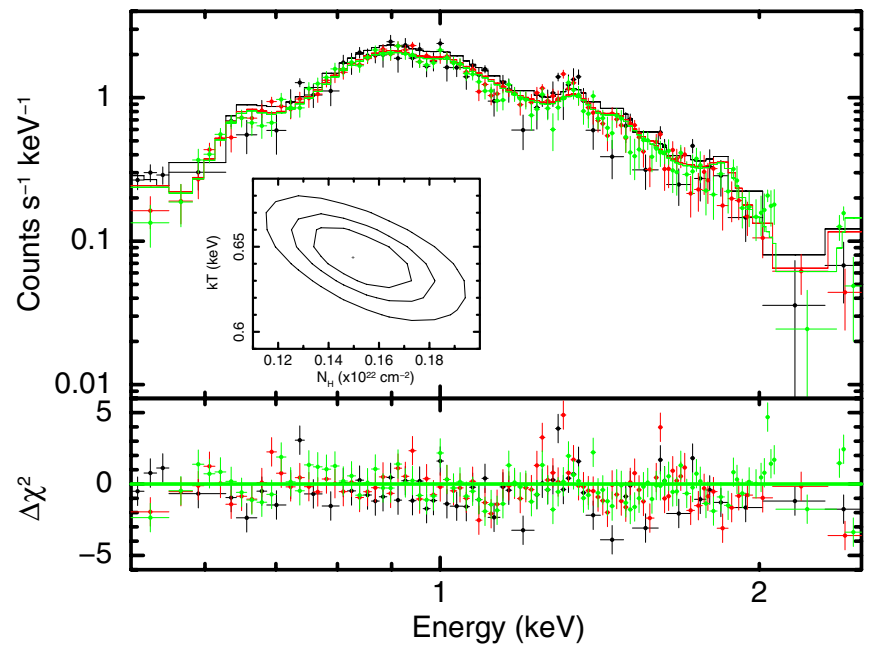

Figure 4. Global X-ray spectra from the three ACIS observations of 30 Doradus (ObsID 5906 in black, ObsID 7263 in red, and ObsID 7264 in green), with the best-fit models overplotted. The inset is the $68 \%, 90 \%$, and $99 \%$ confidence contours for the column density $N_{\mathrm{H}}$ and the temperature $k T_{\mathrm{X}}$. The bottom panel gives the residuals between the data and the model in terms of $\chi^{2}$. We find a best-fit $N_{\mathrm{H}}=1.5_{-0.2}^{+0.3} \times 10^{21} \mathrm{~cm}^{-2}$ and $k T_{\mathrm{X}}=0.64_{-0.02}^{+0.03} \mathrm{keV}$.

the regions $\left(35^{\prime \prime} \approx 8 \mathrm{pc}\right.$ on a side, at a distance $D=50 \mathrm{kpc}$ to the LMC) to match the HST PC image of R136 (Malumuth \& Heap 1994), so that we could use their $L_{\text {bol }}$ value and do not have to resolve the individual point sources in the crowded R136 cluster. The number and position of our 441 regions were 


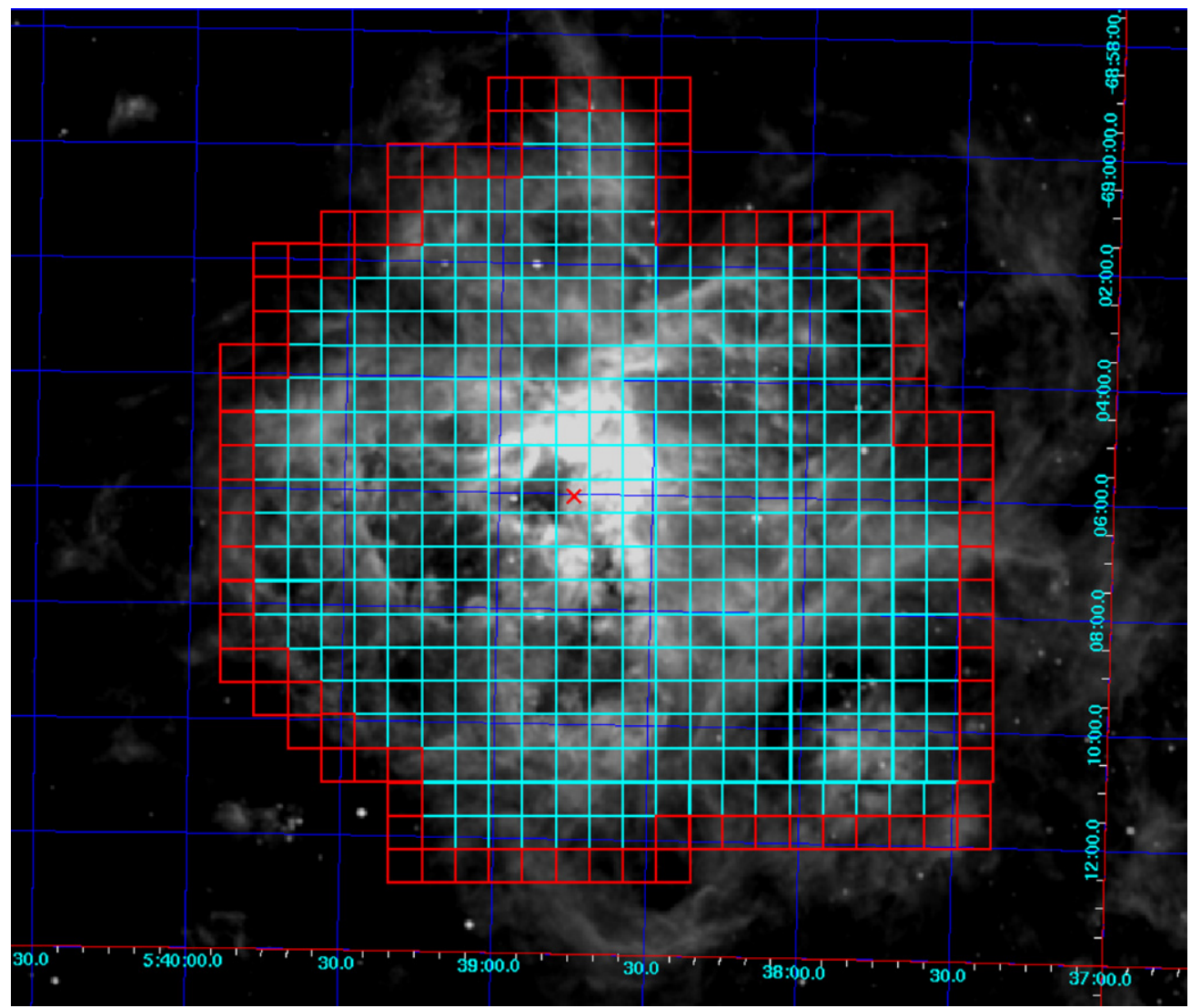

Figure 5. H $\alpha$ image (Smith \& MCELS Team 1998) with the 441 regions we analyzed overplotted. Red squares denote those included in the hot gas leakage analysis of Section 5.1. The red X marks the center of R136.

determined by the field of view and orientation of the $3 \mathrm{~cm}$ radio and Chandra X-ray data. Figure 5 shows the $\mathrm{H} \alpha$ image with all the resulting regions overplotted.

To ascertain the dynamical importance of the feedback processes, we compute the pressures for each region using the methods and relations described below. Since protostellar outflows are only important dynamically in low-mass star clusters (Matzner 2007), we do not expect them to play a big role in 30 Doradus, and we will not consider them in the rest of the paper.

\subsection{Direct Radiation Pressure}

The light output by stars produces a direct radiation pressure that is associated with the photons' energy and momentum. The resulting radiation pressure $P_{\text {dir }}$ at some position within the $\mathrm{H}$ II region is related to the bolometric luminosity of each star $L_{\mathrm{bol}}$ and the distance $r$ the light traveled to reach that point:

$$
P_{\text {dir }}=\sum \frac{L_{\text {bol }}}{4 \pi r^{2} c},
$$

where the summation is over all the stars in the field. In Section 5.2, we describe an alternative definition of radiation pressure and compare the results from each case.

The above relation assumes that the stellar radiation is not attenuated by dust. In Section 3.2, we calculate separately the radiation pressure associated with the light absorbed by dust using Spitzer IR photometry. Given that our results show that $P_{\text {dir }} \gg P_{\mathrm{IR}}$ generally (see Section 4 ), the assumption that the emitted $L_{\mathrm{bol}}$ is unattenuated seems reasonable.

In order to obtain the bolometric luminosities of the massive stars in 30 Doradus, we utilize the UBV photometric data described in Section 2.1. To simplify the calculation, we assume that the bolometric luminosity of R136 obtained by Malumuth \& Heap (1994) originates from the point in the middle of the central region marked with the red $X$ in Figure 5. For the stars located outside R136 within a few arcminutes, the Parker (1993) catalog only includes the apparent $U B V$ magnitudes and colors. Therefore, we follow the procedure outlined by Parker \& Garmany (1993) to obtain absolute bolometric magnitudes of the 1264 stars in the Parker (1993) catalog that are not in the Selman \& Melnick (2005) sample. For the 7697 stars in the Selman \& Melnick (2005) catalog that lie outside the field of the Parker (1993) data, we use their published values for the stars' absolute bolometric magnitudes.

Thus, in total, we calculate the bolometric luminosities $L_{\text {bol }}$ of the R136 cluster and 8961 other stars in 30 Doradus. For each of the 441 regions, we sum these 8962 terms in Equation (1), where $r$ corresponds to the projected distance from the 8962 stars' positions to the region center. In this manner, we compute the radiation pressure "felt" by the 441 regions from all of the starlight in 30 Doradus.

Since the stars are viewed in projection, the actual distance $r$ to a star from the R136 center is observed as a projected distance $\psi$. Therefore, we calculate the direct radiation pressure for two scenarios: one case assuming the stars lie in the same plane $\left(P_{\text {dir }}\right)$ and another case where we attempt to "deproject" the stars positions $\left(P_{\text {dir.3D }}\right)$. Appendix A outlines the procedure we utilize to obtain the deprojected bolometric luminosity of the stars as a function of $r$ and compares $P_{\mathrm{dir}}$ with $P_{\mathrm{dir}, 3 \mathrm{D}}$. We find that $P_{\mathrm{dir}, 3 \mathrm{D}}$ is $10 \%-60 \%$ less than $P_{\text {dir }}$ at radii $\lesssim 20$ pc from 30 Doradus, and the fractional difference between $P_{\text {dir.3D }}$ and $P_{\text {dir }}$ at larger radii is much less $(0.1 \%-3.0 \%)$. As these differences do not affect 


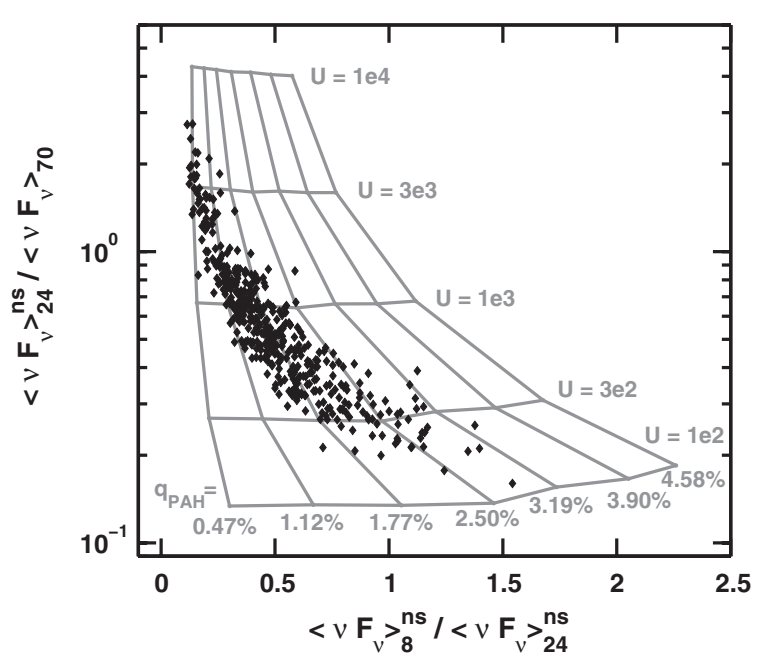

Figure 6. Measured IR flux ratios for the 441 regions (black diamonds) and the predicted flux ratios for different $\mathrm{PAH}$ mass fractions $q_{\mathrm{PAH}}$ and scaling $U$ of the energy density of the dust-processed radiation field (Equation (3)) from DL07. We interpolate the grid of predicted flux ratios to obtain $q_{\mathrm{PAH}}$ and $U$ for each region.

the conclusions of this paper, we will only consider $P_{\text {dir }}$ for the rest of our analyses.

\subsection{Dust-Processed Radiation Pressure}

The stars' radiation will be processed by the nearby dust in the region, and an associated pressure is exerted by the resulting infrared radiation field, $P_{\mathrm{IR}}$. This pressure component could become dynamically important if the expanding $\mathrm{H}_{\text {II }}$ shell is optically thick to the IR light, effectively trapping the radiation inside the H II shell (Krumholz \& Matzner 2009). The pressure of the dust-processed radiation field $P_{\mathrm{IR}}$ can be determined by the energy density of the radiation field absorbed by the dust, $u_{v}$ (i.e., we assume steady state),

$$
P_{\mathrm{IR}}=\frac{1}{3} u_{v}
$$

To find $u_{v}$ in each of our 441 regions, we measure their flux densities $F_{v}$ in the IRAC and MIPS images and compare them to the predictions of the dust models of Draine \& $\mathrm{Li}$ (2007; DL07 hereafter). The DL07 models show how the IR emission spectral energy distribution varies depending on the dust content and the intensity of radiation heating the dust. DL07 assume a mixture of carbonaceous grains and amorphous silicate grains that have a size distribution that reproduces the wavelength-dependent extinction in the local Milky Way (see Weingartner \& Draine 2001). One component of this dust mixture is polycyclic aromatic hydrocarbons (PAHs), smallsized carbonaceous grains that produce strong emission features at $\sim 3-19 \mu \mathrm{m}$ observed in many galaxies.

Since the infrared emission from dust is relatively insensitive to the spectrum of the incident photons with $h v<13.6 \mathrm{eV}, \mathrm{DL} 07$ adopts the spectrum of the local-neighborhood ISM. Then, $u_{v}$ is given by

$$
u_{v}=U u_{v}^{\mathrm{IRSF}},
$$

where $U$ is a dimensionless scale factor and $u_{v}^{\mathrm{IRSF}}$ is the energy density of the $h v<13.6 \mathrm{eV}$ photons in the local ISM, $8.65 \times$ $10^{-13} \mathrm{erg} \mathrm{cm}^{-3}$ (Mathis et al. 1983). We assume that each region is exposed to a single radiation field because the starlight heating

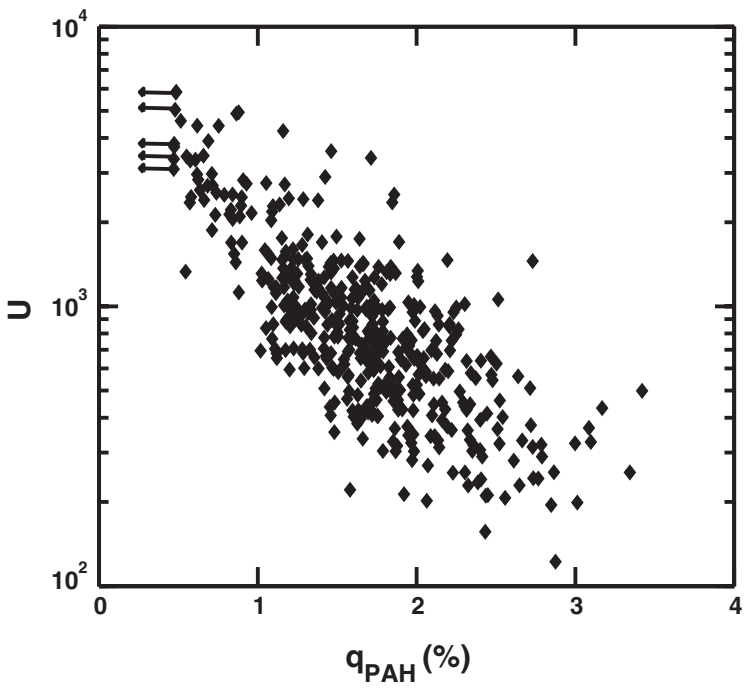

Figure 7. Plot of $U$ vs. PAH fraction $q_{\mathrm{PAH}}$. Arrows reflect upper limits in the $q_{\mathrm{PAH}}$ values, corresponding to the points outside the grid in Figure 6.

the dust comes largely from NGC 2070. In DL07 parameters, this case corresponds to $U_{\min }=U_{\max }$ and $\gamma=0$, where $(1-\gamma)$ is the fraction of the dust mass exposed to the radiation.

For our analyses, we measure the average flux densities $F_{v}$ at 8,24 , and $70 \mu \mathrm{m}$ wavelengths for the 441 regions. We do not consider the $160 \mu \mathrm{m}$ band because its flux density relative to the $70 \mu \mathrm{m}$ is much higher than that is consistent with the DL07 models. We suspect that the $160 \mu \mathrm{m}$ flux is from cold dust that is not associated with 30 Doradus, but is in the sight line to the $\mathrm{H}$ II region.

To ensure that we measure the 8 and $24 \mu \mathrm{m}$ flux densities only from dust and not starlight, we remove the starlight contribution at these wavelengths based on the $3.6 \mu \mathrm{m}$ flux density (which is almost entirely from starlight):

$$
\begin{gathered}
F_{v}^{\mathrm{ns}}(8 \mu \mathrm{m})=F_{v}(8 \mu \mathrm{m})-0.232 F_{v}(3.6 \mu \mathrm{m}) \\
F_{v}^{\mathrm{ns}}(24 \mu \mathrm{m})=F_{v}(24 \mu \mathrm{m})-0.032 F_{v}(3.6 \mu \mathrm{m})
\end{gathered}
$$

where the left-hand sides are the non-stellar flux at the respective wavelengths. The coefficients 0.232 and 0.032 are given in Helou et al. (2004).

To account for the different spatial resolutions of the IR images, we convolved the 3.6, 8, and $24 \mu \mathrm{m}$ images with kernels to match the point-spread function of the $70 \mu \mathrm{m}$ image. For this analysis, we employed the convolution kernels and method described in Section 2.3 of Gordon et al. (2008).

Figure 6 shows the resulting average IR flux ratios, $\left\langle v F_{v}\right\rangle_{24}^{\mathrm{ns}} /\left\langle v F_{v}\right\rangle_{70}$ versus $\left\langle v F_{v}\right\rangle_{8}^{\mathrm{ns}} /\left\langle v F_{v}\right\rangle_{24}^{\mathrm{ns}}$, of our regions. Overplotted are the DL07 model predictions for given values of $q_{\mathrm{PAH}}$, the fraction of dust mass in PAHs, and $U$. Errors in our flux ratios are $\approx 2.8 \%$ from an $\approx 2 \%$ uncertainty in the Spitzer photometry.

We interpolate the $U-q_{\mathrm{PAH}}$ grid using Delaunay triangulation, a technique appropriate for a non-uniform grid, to find the $U$ and $q_{\mathrm{PAH}}$ values for our regions. Figure 7 plots the interpolated values of $U$ versus $q_{\mathrm{PAH}}$. Since the points with the smallest $\left\langle v F_{v}\right\rangle_{8}^{\mathrm{ns}} /\left\langle\nu F_{v}\right\rangle_{24}^{\mathrm{ns}}$ values lie to the left of the $U-q_{\mathrm{PAH}}$ grid in Figure 6, we are only able to set upper limits of $q_{\mathrm{PAH}}=0.47 \%$ for them (marked with arrows in Figure 7). Thus, these regions 


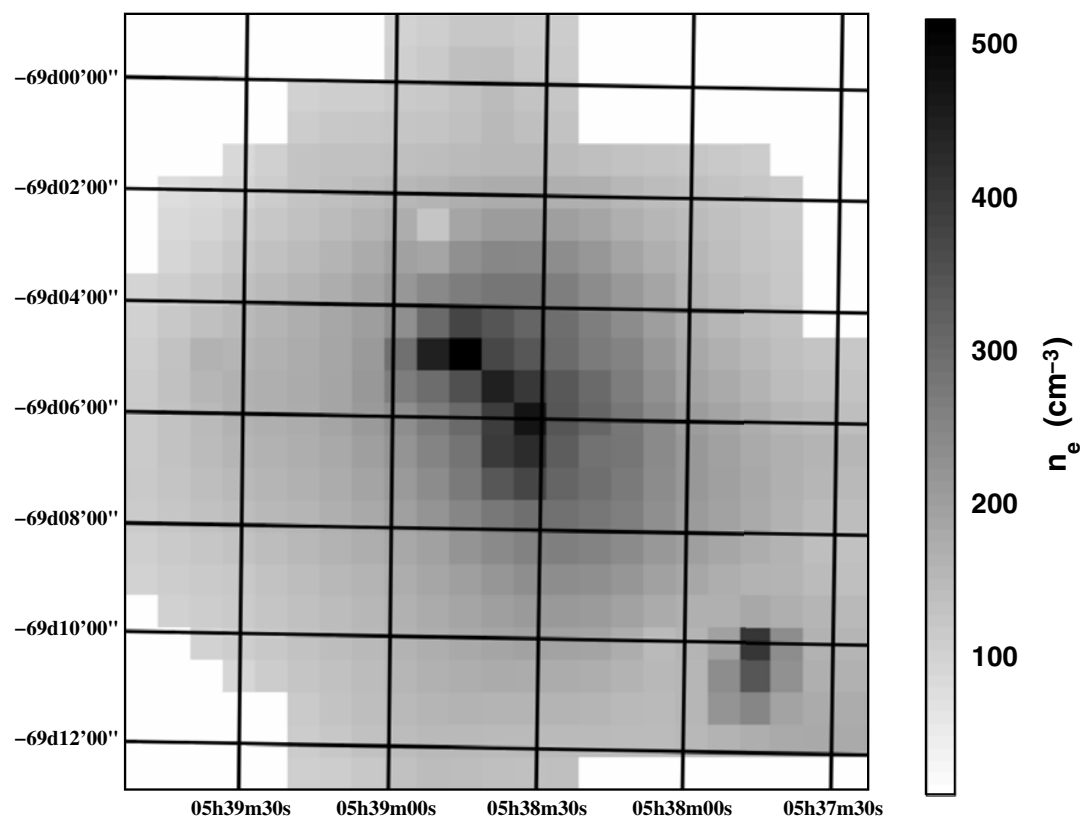

Figure 8. Map of electron density $n_{\mathrm{e}}$ of the warm gas across 30 Doradus, calculated using the bremsstrahlung flux observed in the $3 \mathrm{~cm}$ ATCA data. We find $n_{\mathrm{e}}$ is fairly uniform, with a general range of $n_{\mathrm{e}} \approx 100-500 \mathrm{~cm}^{-3}$.

produce the "wall" of points at $q_{\mathrm{PAH}}=0.47 \%$ in the $U$ versus $q_{\text {PAH }}$ plot.

We find that the PAH fraction spans roughly an order of magnitude, with values up to $q_{\mathrm{PAH}}=3.76 \%$. $U$ (and thus $u_{v}$ ) also varies significantly across 30 Doradus, with $U \approx 91-7640$, corresponding to $u_{v} \approx 6.6 \times 10^{-9}-7.9 \times 10^{-11} \mathrm{erg} \mathrm{cm}^{-3}$. These ranges vary radially, with the largest $U$ and smallest $q_{\mathrm{PAH}}$ close to R136. One possible explanation for the $q_{\mathrm{PAH}}$ radial dependence is that PAHs are destroyed more where the radiation field heating the dust is strong (e.g., Guhathakurta \& Draine 1989). This result is consistent with the analyses of Peeters et al. (2004), who showed that the ratio of PAH to far-IR (dust continuum) emission in Galactic H II regions is inversely correlated with the intensity of the UV field absorbed by the dust.

We utilize the interpolated $U$ values and Equation (3) to obtain the energy density $u_{\nu}$, and thus the pressure of the dustprocessed radiation field in the 441 regions.

\subsection{Warm Ionized Gas Pressure}

Next, we consider the pressure associated with both the warm $\mathrm{H}$ II gas and the hot X-ray gas. The warm ionized gas pressure is given by the ideal gas law, $P_{\mathrm{H}_{\text {II }}}=\left(n_{\mathrm{e}}+n_{\mathrm{H}}+n_{\mathrm{He}}\right) k T_{\mathrm{H} \text { II }}$, where $n_{\mathrm{e}}, n_{\mathrm{H}}$, and $n_{\mathrm{He}}$ are the electron, hydrogen, and helium number densities, respectively, and $T_{\mathrm{H}_{\text {II }}}$ is the temperature of the H II gas, which we assume to be the same for electrons and ions. If helium is singly ionized, then $n_{\mathrm{e}}+n_{\mathrm{H}}+n_{\mathrm{He}} \approx 2 n_{\mathrm{e}}$. The temperature of the H II gas in 30 Doradus is fairly homogeneous, with $T_{\mathrm{H}_{\text {II }}}=10270 \pm 140 \mathrm{~K}$, based on the measurement of $[\mathrm{O}$ III $](\lambda 4959+\lambda 5007) / \lambda 4363$ across 135 positions in the nebula (Krabbe \& Copetti 2002); here, we adopt $T_{\mathrm{H}}$ II $=10^{4} \mathrm{~K}$. Since $T_{\mathrm{H} \text { II }}$ is so uniform, the warm gas pressure is determined by the electron number density $n_{\mathrm{e}}$. We estimate $n_{\mathrm{e}}$ from the average flux density $F_{v}$ of the free-free radio emission in each region (Equation (5.14b), Rybicki \& Lightman 1979):

$$
n_{\mathrm{e}}=\left(\frac{6.8 \times 10^{38} 4 \pi D^{2} F_{\nu} T_{\mathrm{H}}^{1 / 2}}{\bar{g}_{\mathrm{ff}} V}\right)^{1 / 2}
$$

where we have set the Gaunt factor $\bar{g}_{\mathrm{ff}}=1.2$. In the above relation, $D$ is the distance to 30 Doradus (assumed to be $D=$ $50 \mathrm{kpc}$ ) and $V$ is the integrated volume of our regions. For $V$, we assume a radius of the $\mathrm{H}$ II region $R=150 \mathrm{pc}$, and we calculate the volume by multiplying the area of our region squares by the path length through the sphere at the region's position. We measure $F_{v}$ of our regions in the $3.5 \mathrm{~cm}$ ATCA+Parkes image, since bremsstrahlung dominates at that wavelength. Figure 8 shows the resulting map of $n_{\mathrm{e}}$ from these analyses. We find that the central few arcminute area of 30 Doradus has elevated electron densities, with values $n_{\mathrm{e}} \approx 200-500 \mathrm{~cm}^{-3}$; the location of these large electron densities corresponds to the two molecular clouds that form the "ridge" in the center of the nebula (Johansson et al. 1998). The area outside the central $n_{\mathrm{e}}$ enhancement has relatively uniform electron density, with $n_{\mathrm{e}} \approx 100-200 \mathrm{~cm}^{-3}$. In the southwest of 30 Doradus where the supernova remnant (SNR) N157B is located, we obtain elevated $3.5 \mathrm{~cm}$ flux densities, possibly because of a non-thermal contribution from that source. Therefore, the actual $n_{\mathrm{e}}$ may be lower than the values we find in that region.

Our warm gas electron densities are similar to the values obtained by Indebetouw et al. (2009) using Spitzer's Infrared Spectrograph. These authors used the ratio [S III] $\lambda 18.7 \mu \mathrm{m} /$ [S III] $\lambda 33.4 \mu \mathrm{m}$ to map $n_{\mathrm{e}}$ across the central $\approx 2^{\prime}$ of 30 Doradus. They also find enhancements in $n_{\mathrm{e}}$ along the "ridge."

\subsection{Hot Gas Pressure}

The hot X-ray gas arises from shock heating by stellar winds and $\mathrm{SNe}$, and the associated hot gas pressure is given by the relation $P_{\mathrm{X}}=1.9 n_{\mathrm{X}} k T_{\mathrm{X}}$, where $n_{\mathrm{X}}$ and $T_{\mathrm{X}}$ are the electron number density and temperature of the X-ray gas, respectively. The factor of 1.9 arises from the assumption that He is fully ionized and that the He mass fraction is 0.3. As in our warm ionized gas calculation, we assume that the electrons and ions have reached equipartition, so a single temperature describes both. Since the hot gas can exist over a range of $T_{\mathrm{X}}$, we measure both $n_{\mathrm{X}}$ and $T_{\mathrm{X}}$ by modeling the bremsstrahlung spectrum at $\mathrm{X}$-ray wavelengths. 


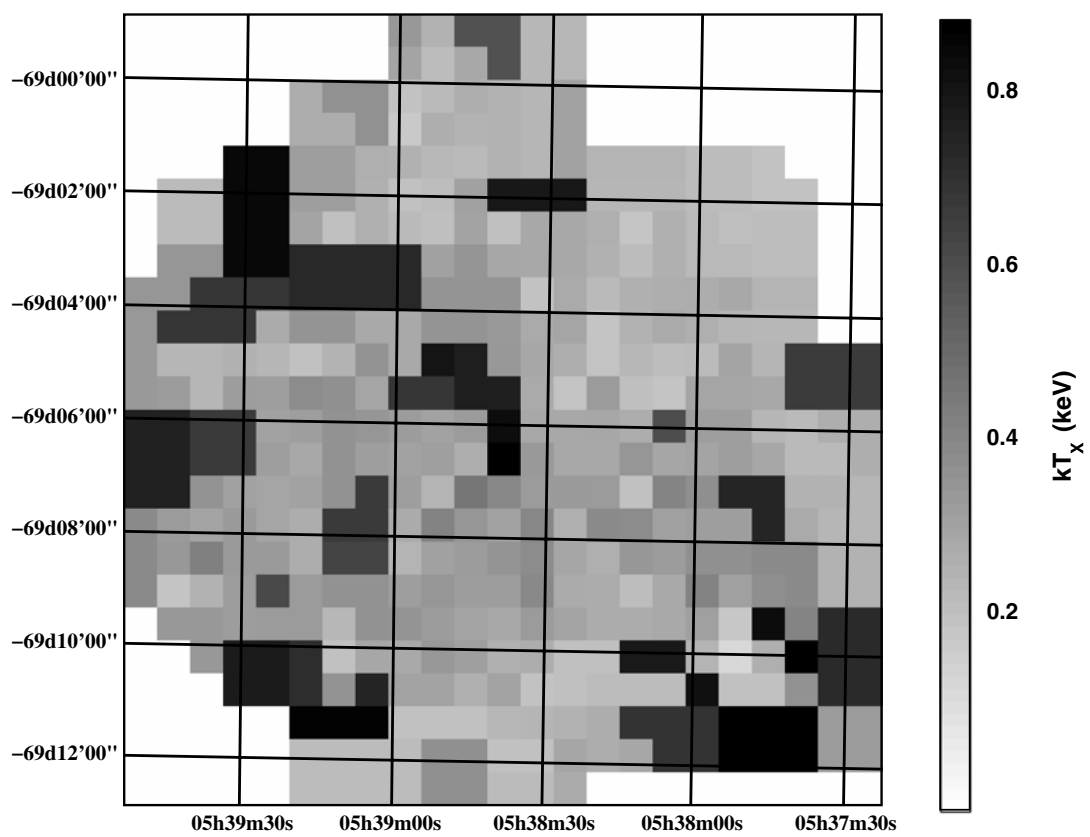

Figure 9. Map of the $\mathrm{X}$-ray gas temperature $k T_{\mathrm{X}}$ (in $\mathrm{keV}$ ) across 30 Doradus. These values were obtained by modeling the Chandra $\mathrm{X}$-ray spectra from each region.

From the three Chandra observations, we extracted Chandra $\mathrm{X}$-ray spectra from each region using the CIAO command specextract. Background spectra were also produced from a circular region of radius $\approx 15^{\prime \prime}$ that is $\approx 2^{\prime}$ east of 30 Doradus (the cyan circle in Figure 3), and these were subtracted from the source spectra. The resulting spectra were fit using XSPEC Version 12.4.0. Data were grouped such that a minimum of five counts were in each energy bin, and the spectra from the three ACIS observations of a given region were fit simultaneously to improve statistics (i.e., they were fit jointly, with more weight given to the data from the longer integrations). Around the edges of the $\mathrm{H}$ II region, the $\mathrm{X}$-ray signal is weaker, so we combined adjacent regions to achieve sufficient counts for an accurate fit.

Spectra were modeled as an absorbed hot diffuse gas in collisional ionization equilibrium using the XSPEC components phabs and mekal (Mewe et al. 1985, 1986; Liedahl et al. 1995). In this fit, we assumed a metallicity $Z \sim 0.5 Z_{\odot}$, the value measured in H II regions in the LMC (Kurt \& Dufour 1998). For regions in the southwest of 30 Doradus with strong emission from the SNR N157B, we added a power-law component to account for the non-thermal emission from the SNR. We obtained good fits statistically, with reduced chi-squared values of $0.80-1.30$ with $60-300$ dof. If a region's fit had reduced chi-squared values outside this range or less than 60 dof, we combined its spectra with those of adjacent regions to increase the signal. The latter criterion was selected since we found generally that the shape of the bremsstrahlung continuum was not discernable with less than 60 dof.

From our fits, we can estimate the electron number density $n_{\mathrm{X}}$ of each region based on the emission measure EM of our models. Emission measure is defined as $\mathrm{EM}=\int n_{\mathrm{X}}^{2} d V$. Thus,

$$
n_{\mathrm{X}}=\left(\frac{\mathrm{EM}}{V}\right)^{1 / 2}
$$

where $V$ is the integrated volume of our region (the same as used in Equation (6)). Since we are interested in the contribution of the X-ray pressure to the global dynamics, we have divided the EM by the integrated volume of a region $V$ in calculating $n_{\mathrm{X}}$, rather than the volume occupied by the hot gas. In the former case, the density $n_{\mathrm{X}}$ goes as the filling factor $f^{-1 / 2}$; in the latter scenario, $n_{\mathrm{X}} \propto f \cdot f^{-1 / 2}=f^{1 / 2}$. If the filling factor of the hot gas is small, the thermal pressure of the bubbles may be high internally; however, the hot gas would be insignificant dynamically because it occupies a negligible volume and thus exerts little pressure on the material that bounds the $\mathrm{H}$ II region. Therefore, for our purposes of assessing the dynamical role of the hot gas, it is appropriate to use the integrated volume in calculating $n_{\mathrm{X}}$.

Figure 9 shows a map of the best-fit temperatures $k T_{\mathrm{X}}$ from the spectral modeling analyses. The $\mathrm{X}$-ray gas temperatures are elevated in several areas, including in the southwest (the bottom right of Figure 9), where the SNR N157B is located, and at the center near R136. Figure 10 gives the map of the hot gas electron density across 30 Doradus from our fits. We find a mean $\left\langle n_{\mathrm{X}}\right\rangle=0.12 \mathrm{~cm}^{-3}$. The hot gas electron density is much less than that of the warm gas since many fewer electrons are heated to X-ray emitting temperatures $\left(\sim 10^{7} \mathrm{~K}\right)$ than to the moderate temperatures $\sim 10^{4} \mathrm{~K}$ of the warm gas.

\section{RESULTS}

Following the multi-wavelength analyses and methodology of Section 3, we calculate the pressures associated with the direct stellar radiation pressure $P_{\mathrm{dir}}$, the dust-processed radiation pressure $P_{\mathrm{IR}}$, the warm ionized gas pressure $P_{\mathrm{H}}$ II and the hot $\mathrm{X}$-ray gas pressure $P_{\mathrm{X}}$. Figure 11 plots the results as a function of the distance from the center of R136; data of similar radii (defined as radii within $10 \%$ fractionally of each other) are binned to simplify the plot and to make trends more readily apparent. By comparing the radial trends of the different components, we find that $P_{\text {dir }}$ dominates at distances $\lesssim 75$ pc from R136, while $P_{\mathrm{H} \text { II }}$ dominates at larger radii from R136. Additionally, $P_{\mathrm{IR}}$ and $P_{\mathrm{X}}$ do not appear to contribute significantly, although they are on the order of $P_{\text {dir }}$ at distances $\gtrsim 100 \mathrm{pc}$ from R136.

As demonstrated in Figure 11, we find that $P_{\mathrm{H} \text { II }}>P_{\mathrm{X}}$. The lack of pressure balance between these two components is 


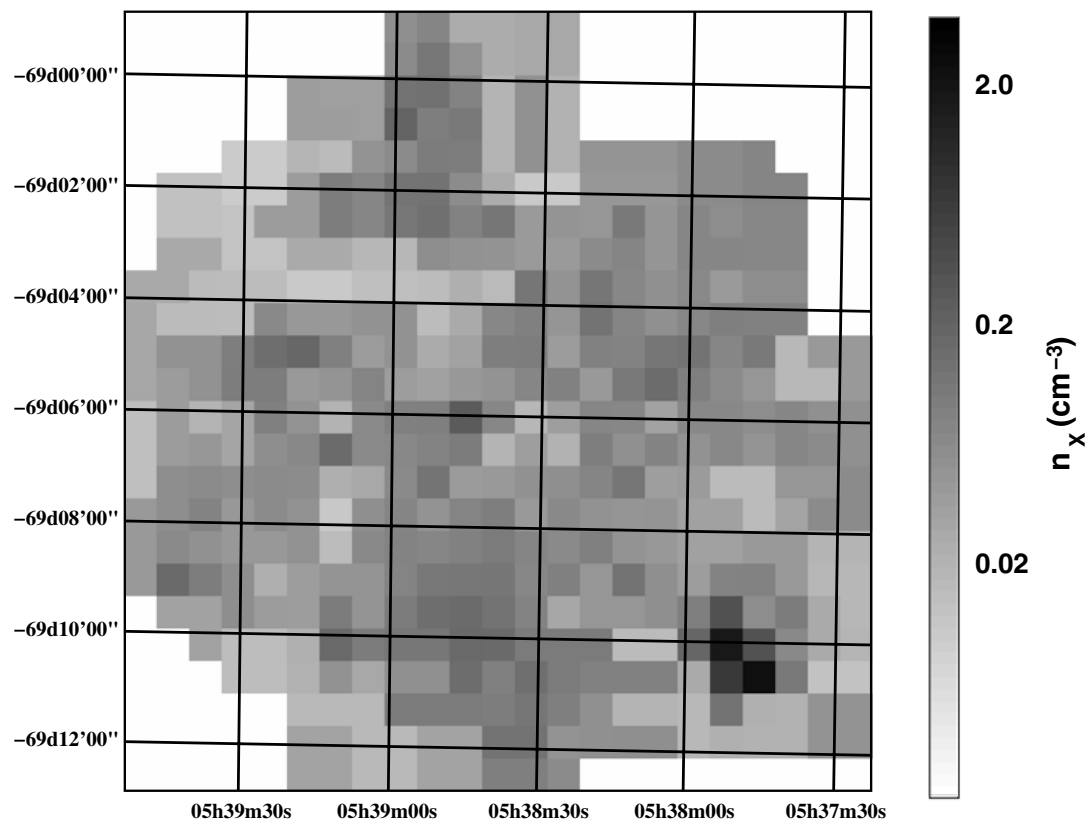

Figure 10. Map of the hot gas electron density $n_{\mathrm{X}}$ (in particles $\mathrm{cm}^{-3}$ ) across 30 Doradus. These values were obtained by modeling the Chandra X-ray spectra from each region, which output the best-fit EM. We converted EM to $n_{\mathrm{X}}$ using Equation (7).

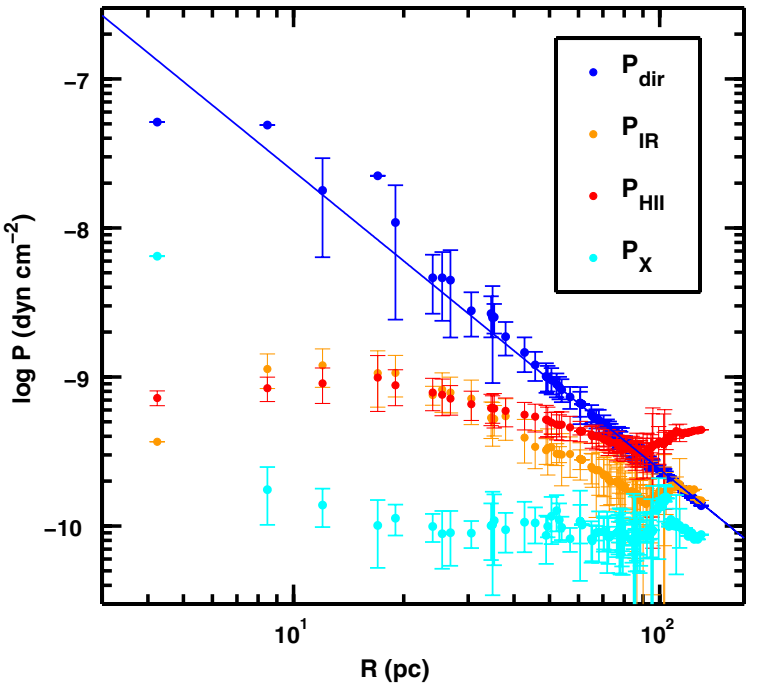

Figure 11. All pressures vs. radius from the center of R136. Regions with similar radii (defined as radii within $10 \%$ fractionally of each other) are binned to simplify the plot and make trends more readily apparent, and bars reflect the $1 \sigma$ standard deviations in the pressures at the given radii. Generally, $P_{\mathrm{dir}}$ dominates at radii $\left\langle 75 \mathrm{pc}\right.$ and follows a $P_{\mathrm{dir}} \propto r^{-2}$ relation (the blue solid line), whereas $P_{\mathrm{H} \text { II }}$ dominates at larger distances from R136. $P_{\mathrm{IR}}$ and $P_{\mathrm{X}}$ do not appear to contribute significantly.

consistent with our finding (see Section 5.1) that the X-ray gas does not remain adiabatic and trapped inside the shell. Instead, the hot gas is either leaking out or is mixing with cool gas and suffering rapid radiative losses as a result. In either case, the hot gas is likely to be flowing at a bulk speed comparable to its sound speed, and thus it will not have time to reach pressure equilibrium with the cooler gas that surrounds it before escaping the $\mathrm{H}$ II region. Alternatively, it may be that pressure balance is established between the warm ionized gas and the ram pressure of the hot gas, whereas we have only measured the thermal pressure. This picture is consistent with the anticoincidence of the warm and hot gas noted by previous X-ray work (e.g., Wang 1999; Townsley et al. 2006a).
In Figure 12, we give the maps of the four pressures across 30 Doradus for our 441 regions. $P_{\text {dir }}$ has a smooth profile due to its $1 / r^{2}$ dependence, while $P_{\mathrm{H}}$ iI is fairly uniform across 30 Doradus (as expected for a classical $\mathrm{H}$ II region). Compared to those components, $P_{\mathrm{IR}}$ and $P_{\mathrm{X}}$ have more variation throughout the source. Additionally, all the maps have significant enhancements in the central regions near R136; in the cases of $P_{\mathrm{IR}}$ and $P_{\mathrm{H} \text { II }}$, the elevated pressures correspond to the molecular "ridge" in 30 Doradus (as seen in the CO contours in Figure 2). Additionally, all except $P_{\text {dir }}$ have greater pressures in the regions near the SNR N157B (the bottom right of the maps).

We can utilize the obtained pressures to estimate the total energy of each component. In particular, we measure the total energy density $u$ in a given radius bin of Figure 11 and multiply by the volume of its shell (where we have set the shell thickness to the difference of the upper and lower bound radius of that bin). We convert pressures $P$ to energy densities $u$ using the relations $P_{\mathrm{dir}}=u_{\mathrm{dir}}, P_{\mathrm{IR}}=\frac{1}{3} u_{\mathrm{IR}}, P_{\mathrm{H} \text { II }}=\frac{2}{3} u_{\mathrm{HII}}$, and $P_{\mathrm{X}}=\frac{2}{3} u_{\mathrm{X}}$. Using this approach, we find the following total energies for each component: $E_{\mathrm{dir}}=5.1 \times 10^{53} \mathrm{erg}$, $E_{\mathrm{IR}}=1.7 \times 10^{53} \mathrm{erg}, E_{\mathrm{H} \text { II }}=2.8 \times 10^{53} \mathrm{erg}$, and $E_{\mathrm{X}}=$ $6.5 \times 10^{52} \mathrm{erg}$. Therefore, the direct and dust-processed radiation fields and the warm ionized gas contribute similarly to the energetics of the region, and every component is $\gtrsim 2$ orders of magnitude above the typical kinetic energy of a single SN explosion.

\section{DISCUSSION}

\subsection{Leakage of the Hot Gas}

As mentioned previously, the X-ray emission in 30 Doradus arises from the shock heating of gas to temperatures of $\sim 10^{7} \mathrm{~K}$ by stellar winds and SNe. These feedback processes eventually carve out large cavities, called bubbles and superbubbles, filled with diffuse X-ray emission. In Figure 11, we demonstrated that the pressure associated with the hot gas $P_{\mathrm{X}}$ is comparatively low relative to the other pressure components. Here, we explore the implications of this result in regard to the trapping/leakage of 

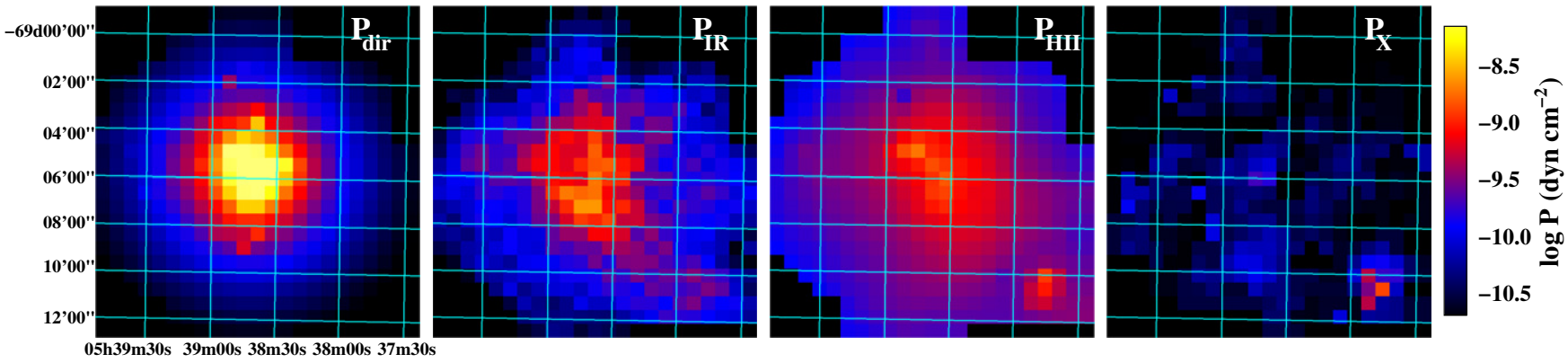

Figure 12. Maps of the four pressure components across 30 Doradus. All four are on the same color scale to enable visual comparison. Consistent with Figure $11, P_{\mathrm{dir}}$ dominates in the central few arcminutes, while the $P_{\mathrm{H} \text { II }}$ dominates at larger distances from R136.

the hot gas. For this discussion, we will consider stellar winds only and ignore the contribution by $\mathrm{SNe}$; this assumption is reasonable given that the mechanical energy of one $\mathrm{SN}$ is on the order of the amount injected by winds over a single massive star's lifetime (Castor et al. 1975). This assumption is valid at the $0.5 Z_{\odot}$ of the LMC: simulations of a $5.5 \times 10^{4} M_{\odot}$ star cluster in Starburst99 (Leitherer et al. 1999) showed that the total wind luminosity decreased by roughly a factor of two from the solar to half-solar metallicity case.

There are several competing theoretical models to account for the X-ray luminosity in bubbles and superbubbles. The models of Castor et al. (1975) and Weaver et al. (1977) assume that the shock-heated gas is completely confined by a cool shell expanding into a uniform density ISM. An alternative theory proposed by Chevalier \& Clegg (1985) ignores the surrounding ISM and employs a steady-state, free-flowing wind. Recently, Harper-Clark \& Murray (2009) introduced an intermediate model between these two, whereby the ambient ISM is nonuniform. In this case, only some of the hot gas can escape freely through the holes in the shell.

The fraction of hot gas confined by the shell directly determines the hot gas pressure on the shell as well as the X-ray luminosity within the bubble. If the shell is very porous, the shock-heated gas will escape easily, the wind energy will be lost from the bubble, and the associated pressure and luminosity will be low. By comparison, a more uniform shell will trap the hot gas, retain the wind energy within the bubble, and the corresponding X-ray pressure and luminosity will be much greater. As such, in the latter case, the shocked winds could have a significant role in the dynamics of the $\mathrm{H}$ II region. We note that the warm gas is not able to leak similarly because its sound speed is less than the velocities of the shells $\left(20-200 \mathrm{~km}^{-1}\right.$; Chu \& Kennicutt 1994).

To assess whether the hot gas is trapped inside the shell and is dynamically important, we measure the ratio of the hot gas pressure to the direct radiation pressure, $f_{\text {trap }, \mathrm{X}}=P_{\mathrm{X}} / P_{\mathrm{dir}}$, and compare it to what $f_{\text {trap, } \mathrm{X}}$ would be if all the wind energy was confined. We can calculate the trapped-wind value using the wind-luminosity relation (Kudritzki et al. 1999; Repolust et al. 2004), which indicates that the momentum flux carried by winds from a star cluster is about half that carried by the radiation field if the cluster samples the entire IMF. Written quantitatively, $0.5 L_{\mathrm{bol}} / c=\dot{M}_{\mathrm{w}} v_{\mathrm{w}}$, where $\dot{M}_{\mathrm{w}}$ is the mass flux from the winds that launched at a velocity $v_{\mathrm{w}}$. The mechanical energy loss $L_{\mathrm{w}}$ of the winds is then given by

$$
L_{\mathrm{w}}=\frac{1}{2} \dot{M}_{\mathrm{w}} v_{\mathrm{w}}^{2}=\frac{L_{\mathrm{bol}}^{2}}{8 \dot{M}_{\mathrm{w}} c^{2}},
$$

and the mechanical energy of the winds is simply $E_{\mathrm{w}}=L_{\mathrm{w}} t$, where $t$ is the time since the winds were launched. Putting these relations together, the trapped $\mathrm{X}$-ray gas pressure $P_{\mathrm{X}, \mathrm{T}}$ is

$$
P_{\mathrm{X}, \mathrm{T}}=\frac{2 E_{\mathrm{W}}}{3 V_{\mathrm{H} \text { II }}}=\frac{L_{\mathrm{bol}}^{2} t}{16 \pi \dot{M}_{\mathrm{W}} c^{2} R_{\mathrm{H} \text { II }}^{3}},
$$

where $V_{\mathrm{H} \text { II }}$ is the volume of the H II region.

Given that $P_{\mathrm{dir}}=L_{\mathrm{bol}} /\left(4 \pi R_{\mathrm{H} \text { II }}^{2} c\right)$, then $f_{\text {trap, } \mathrm{X}}$ is

$$
f_{\text {trap }, \mathrm{X}}=\frac{L_{\mathrm{bol}} t}{4 \dot{M}_{\mathrm{W}} c R_{\mathrm{H} \text { II }}}=\frac{L_{\mathrm{bol}}}{4 \dot{M}_{\mathrm{w}} c v_{\mathrm{sh}}},
$$

where we have set $R_{\mathrm{H}} / t=v_{\mathrm{sh}}$, the velocity of the expanding shell. Finally, we put $\dot{M}_{\mathrm{w}}$ in terms of $L_{\mathrm{bol}}$ and $v_{\mathrm{w}}$, so that Equation (10) reduces to

$$
f_{\text {trap }, X}=\frac{v_{\mathrm{w}}}{2 v_{\mathrm{sh}}} .
$$

We use the above equation to obtain an order-of-magnitude estimate of $f_{\text {trap, } X}$ if all the wind energy is confined by the shell. We assume a wind velocity $v_{\mathrm{w}} \sim 1000 \mathrm{~km} \mathrm{~s}^{-1}$ (the escape velocity from an $\mathrm{O} 6 \mathrm{~V}$ star; a reasonable order-ofmagnitude estimate, since $\mathrm{O} 3$ stellar winds are faster and WR winds would be slower than this value). If we set $v_{\text {sh }} \sim$ $25 \mathrm{~km} \mathrm{~s}^{-1}$ (the expansion velocity over 30 Doradus given by optical spectroscopy; Chu \& Kennicutt 1994), then $f_{\text {trap, } X} \sim 20$.

We can compare this $f_{\text {trap, } \mathrm{X}}$ to our observed values for the regions closest to the shell (the ones along the rim of our 441 squares in Figure 5); Figure 13 shows the histogram of our observed $f_{\text {trap }, \mathrm{X}}$ values. We find a mean and median $f_{\text {trap, } \mathrm{X}}$ of 0.30 and 0.27 , respectively, for our outermost regions. Over 30 Doradus, the highest values of $f_{\text {trap, } \mathrm{X}}$ are near the SNR N157B in the southwest corner of 30 Doradus (see Figure 14), where the hot gas is being generated and has not had time to vent. Other locations where $f_{\text {trap,X }}$ is elevated are regions with strong $\mathrm{X}$-ray emission and weak $\mathrm{H} \alpha$ emission. Morphologically, these areas could be where the hot gas is blowing out the 30 Doradus shell.

The observed $f_{\text {trap, } \mathrm{X}}$ values are 1-2 orders of magnitude below what they would be if the wind was fully confined. As a consequence, we find that $P_{\mathrm{X}}$ of our regions is too low to be completely trapped in the $\mathrm{H}$ II region (the Castor et al. model), and the X-ray gas must be leaking through pores in the shell. This result is consistent with the Harper-Clark \& Murray model of partial confinement of the hot gas, and the weakness of $P_{\mathrm{X}}$ relative to $P_{\text {dir }}$ suggests that the hot gas does not play a significant role in the dynamics of the $\mathrm{H}$ II region.

We note here that our rim regions in this analysis are $\sim 70-130$ pc from R136, which is less than the estimated radius 


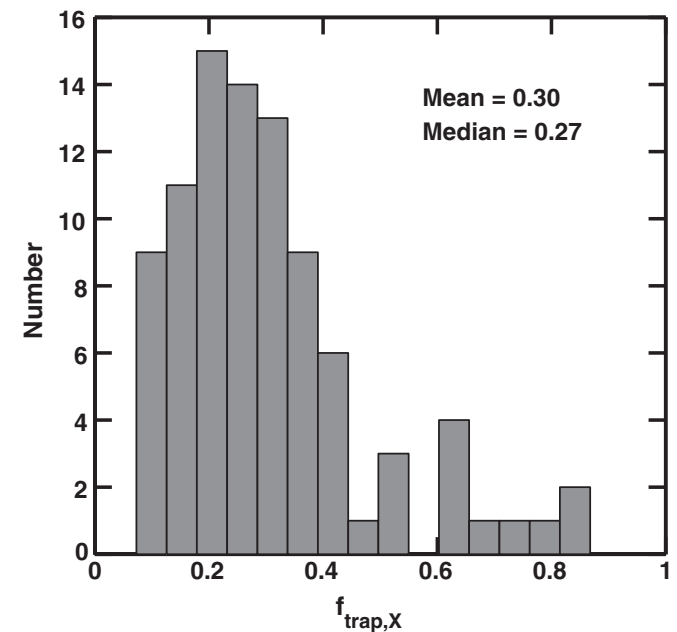

Figure 13. Histogram of $f_{\text {trap }, \mathrm{X}}=P_{\mathrm{X}} / P_{\mathrm{dir}}$, the ratio of the hot gas pressure to the direct radiation pressure, for the regions that are along the rim of our 441 squares in Figure 5. We find that the mean $f_{\text {trap, } X}$ is 0.30 and the median is 0.27 , far below the values expected if the hot gas is completely confined in 30 Doradus $\left(f_{\text {trap }, X} \sim 20\right.$; see the text). This result is evidence that the hot gas is leaking out of the shell.

of $R_{\mathrm{H} \text { II }}=110-185$ pc. Therefore, our $f_{\text {trap,X }}$ values are lower limits, and the true $f_{\text {trap }, \mathrm{X}}$ at the shell may be greater by a factor of a few. Nonetheless, the conclusions would remain the same.

An alternative explanation for the weak X-ray luminosity is that the hot gas mixes with the cool gas, and the hot gas temperature is lowered enough so that the gas can cool efficiently. In that case, the energy is still lost from the system, via radiative cooling instead of the escape of the X-ray emitting material. Far ultraviolet spectroscopy is necessary to determine the level of mixing between the gas components.

\subsection{On the Definition of Radiation Pressure}

In this paper, we have defined the radiation pressure as related to the energy and momentum flux of the light radiated by the stars in 30 Doradus. Alternatively, the radiation pressure could be characterized as the force per unit area exerted by the radiation on matter. The two cases produce divergent results regarding the radial dependence of $P_{\mathrm{dir}}$. In particular, the former case has large $P_{\text {dir }}$ close to the star cluster and a decline in $P_{\text {dir }}$ with distance from the center. By contrast, the latter predicts small $P_{\text {dir }}$ in the H II-region interior, and $P_{\text {dir }}$ becomes significant near the neutral shell where the radiation will be absorbed (see Appendix B).

Each definition of radiation pressure reveals distinct information about an $\mathrm{H}$ il region. When considering the global dynamics of expansion of an $\mathrm{H}$ II region, it is necessary to characterize $P_{\mathrm{dir}}$ as the energy density of the radiation field, since that definition reflects the total energy and momentum budget available to drive motion. Alternatively, measurement of the force exerted by radiation on matter facilitates a local estimate of the internal density distribution of an $\mathrm{HII}$ region. As we are interested principally on the dynamical role of radiation pressure, we have adopted the former definition of $P_{\mathrm{dir}}$ in this paper.

\subsection{H II Region Dynamics}

In Section 4, we found that the direct radiation pressure $P_{\text {dir }}$ dominates over the ionized gas pressure $P_{\mathrm{H} \text { II }}$ at radii $\lesssim 75 \mathrm{pc}$,

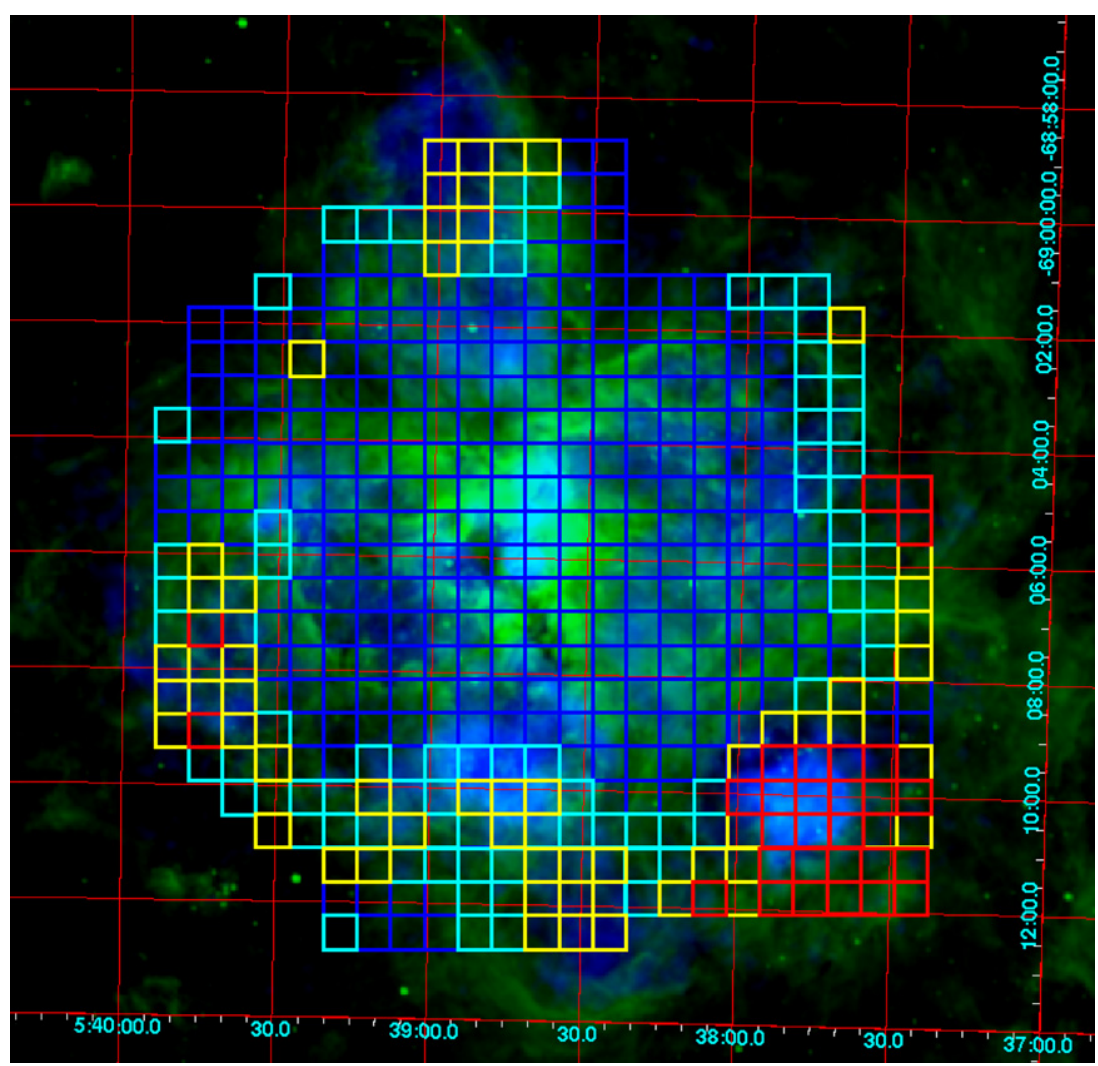

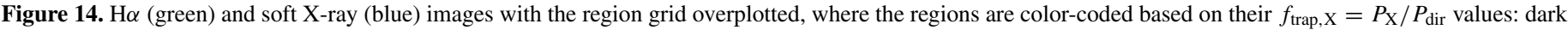

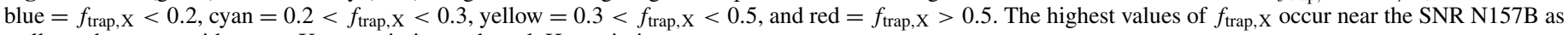
well as other areas with strong $\mathrm{X}$-ray emission and weak $\mathrm{H} \alpha$ emission. 
implying that the radiation has played a role in the dynamics in 30 Doradus. Significant radiation pressure alters the properties of an H II region (e.g., the density profile: Draine 2010) and causes the expansion to proceed differently than in a classical $\mathrm{H}$ II region with ionized gas-driven expansion. In particular, Krumholz \& Matzner (2009) demonstrated that radiation-driven expansion imparts more momentum to the shell, accelerating the expansion at early times relative to that of gas-driven expansion. Indeed, an additional force must have dominated at early times in 30 Doradus since the shell velocity $v_{\mathrm{sh}} \sim 25 \mathrm{~km} \mathrm{~s}^{-1}$ (Chu \& Kennicutt 1994) is too fast to have been gas-driven alone because the H II gas sound speed is $c_{\mathrm{s}} \approx 10 \mathrm{~km} \mathrm{~s}^{-1}$.

To determine the characteristic radius $r_{\mathrm{ch}}$ where the $\mathrm{HII}$ region shell transitioned from radiation pressure driven to gas pressure driven, we can set these pressure terms at the shell equal and solve for $r_{\mathrm{ch}}$. Broadly, the pressures at the shell have different dependences with the shell radius $r_{\mathrm{H} \text { II }}: P_{\mathrm{dir}} \propto r_{\mathrm{HII}}^{-2}$ and $P_{\mathrm{H} \text { II }} \propto r_{\mathrm{HII}}^{-3 / 2}$. Setting them equal and solving for $r_{\mathrm{ch}}$ (Equation (4) in Krumholz \& Matzner 2009, the "embedded case"), we find

$$
r_{\mathrm{ch}}=\frac{\alpha_{\mathrm{B}}}{12 \pi \phi}\left(\frac{\epsilon_{0}}{2.2 k_{\mathrm{B}} T_{\mathrm{H} \text { II }}}\right)^{2} f_{\text {trap }, \text { tot }}^{2} \frac{\psi^{2} S}{c^{2}},
$$

where $\alpha_{\mathrm{B}}$ is the case-B recombination coefficient and $\epsilon_{0}=$ $13.6 \mathrm{eV}$, the photon energy necessary to ionize hydrogen. The dimensionless quantity $\phi$ accounts for dust absorption of ionizing photons and for free electrons from elements besides hydrogen; $\phi=0.73$ if He is singly ionized and $27 \%$ of photons are absorbed by dust (typical for a gas-pressure-dominated H II region; McKee \& Williams 1997). The $f_{\text {trap,tot }}$ represents the factor by which radiation pressure is enhanced by trapping energy in the shell through several mechanisms; the trapped hot gas $f_{\text {trap, } \mathrm{X}}$ calculated in Section 5.1 is one component that can contribute to $f_{\text {trap,tot }}$ (as discussed in Section 5.1). Here, we adopt $f_{\text {trap,tot }}=2$, as in Krumholz \& Matzner (2009). Finally, $\psi$ is the ratio of the bolometric power to the ionizing power in a cluster; we set $\psi=3.2$ using the $\langle S\rangle /\left\langle M_{*}\right\rangle$ and the $\langle L\rangle /\left\langle M_{*}\right\rangle$ relations of Murray \& Rahman (2010).

Putting all these terms together, we find $r_{\mathrm{ch}} \approx 33 \mathrm{pc}$. Physically, this result means that early in the expansion before it reached a radius of $33 \mathrm{pc}, 30$ Doradus dynamics could have been radiation pressure dominated, and it has since become gas pressure dominated. Alternatively, it is possible that the hot gas pressure dominated at early times and has become weaker as the $\mathrm{H}$ II region expands.

The radiation-driven or hot gas-driven expansion at early times in 30 Doradus would have facilitated the expulsion of gas from the central star cluster. In particular, since the warm gas sound speed $\left(\sim 10 \mathrm{~km} \mathrm{~s}^{-1}\right)$ is less than the escape velocity of R136 $\left(\sim 20 \mathrm{~km} \mathrm{~s}^{-1}\right.$, given a mass $M=5.5 \times 10^{4} M_{\odot}$ in a radius $R=1 \mathrm{pc}$; Hunter et al. 1995), an alternative mechanism is necessary to remove the gas and regulate star formation (e.g., Krumholz \& Matzner 2009; Fall et al. 2010). We conclude that the radiation pressure or hot gas pressure likely played this role in 30 Doradus, decreasing the available mass to make new stars and slowing star formation in the region.

\section{SUMMARY}

In this paper, we have utilized multi-wavelength (radio, infrared, optical/UV, and X-ray) imaging to assess the role of several stellar feedback mechanisms in the giant $\mathrm{H}$ II region
30 Doradus in the LMC. In particular, we have measured observationally the pressures associated with possible sources of energy and momentum to drive the dynamics of the region: the direct radiation from stars, the dust-processed infrared radiation field, the warm ionized gas from massive stars, and the hot gas shock-heated by stellar winds and SNe. We have exploited the high-resolution images of 30 Doradus to map these pressure components in 441 square regions, with dimensions of $35^{\prime \prime} \times 35^{\prime \prime}$. We have found that the direct radiation pressure from stars dominates at distances less than $75 \mathrm{pc}$ from the central star cluster, whereas the warm ionized gas pressure dominates at larger radii. By contrast, the hot gas pressure and the dust-processed radiation pressure do not contribute significantly, indicating that these components are not dynamically important. However, we cannot rule out that the hot gas pressure dominated at early times has become weaker with the $\mathrm{H}$ II region expansion.

We have discussed two implications of our results: the partial confinement of the hot gas and the dynamical role of radiation pressure in 30 Doradus. First, the weakness of the X-ray gas pressure relative to the direct radiation pressure suggests that the hot gas is only partially confined and is leaking out of the pores in the H II shell. Second, the significant radiation pressure near the star cluster indicates that radiation pressure may have driven the expansion of the $\mathrm{H}$ II shell at early times. This result suggests observationally that radiation pressure may be dynamically important in massive star clusters, reinforcing that radiation pressure is a viable mechanism to remove gas from $\mathrm{H}$ II regions and to regulate star formation. Indeed, if NGC 2070 was more massive, the radiation pressure could even expel gas at high enough velocities to launch a galactic wind (Murray et al. 2010a).

The work presented here is a first step to measure observationally the relative role of stellar feedback mechanisms in star-forming regions. Although we have applied our techniques to one source, 30 Doradus, our methods to extract dynamical information from multi-wavelength images can be applied to other sources as well. Consequently, we plan to perform these analyses on all the $\mathrm{H}$ II regions in the LMC with available data to develop a broad observational understanding of these stellar feedback mechanisms and their role in regulating star formation.

We thank Bruce Draine, Xander Tielens, and Fernando Selman for helpful discussions. We also thank Jasmina Lazendic-Galloway for generously providing the $3.5 \mathrm{~cm}$ ATCA image of 30 Doradus. This work is supported by an AAUW American Dissertation Fellowship (L.A.L.) and by the National Science Foundation through grant NSF-AST0955836. M.R.K. acknowledges support from an Alfred P. Sloan Fellowship; NASA through ATFP grant NNX09AK31G; NASA as part of the Spitzer Theoretical Research Program, through a contract issued by the JPL; and the National Science Foundation through grant AST-0807739.

\section{APPENDIX A}

\section{DEPROJECTING THE STARS IN 30 DORADUS}

Since the stars are viewed in projection, the actual distance $r$ to a star from the R136 center is observed as a projected distance $\psi$ (see Figure 15). Therefore, we calculate the direct radiation pressure for two scenarios: one case assuming the stars lie in the same plane (i.e., $r=\psi ; P_{\text {dir }}$ ) and another case where we 

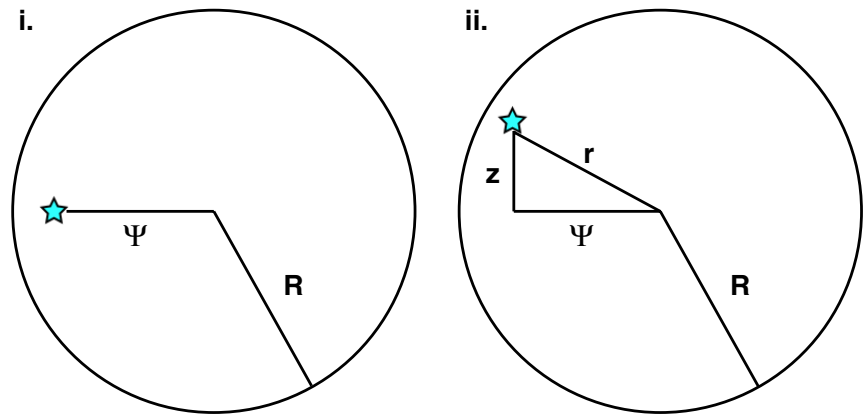

Figure 15. Diagram explaining how projection effects may lead to erroneous measurement of the actual distance $r$ of a star to the star cluster center. (a) Face-on view of an $\mathrm{H}$ II region of radius $R$. The projected distance from the star to the center is $\psi$. (b) View from above of the same $\mathrm{H}$ II region. In this case, it is apparent that the star does not lie in the midplane of the sphere, and the actual distance is $r=\sqrt{\psi^{2}+z^{2}}$, where $z$ is the line-of-sight distance to the star from the sphere's midplane.

(A color version of this figure is available in the online journal.)

attempt to "deproject" the stars positions (i.e., $r=\sqrt{\psi^{2}+z^{2}}$, where $z$ is the line-of-sight distance to the star from the sphere's midplane; $\left.P_{\mathrm{dir}, 3 \mathrm{D}}\right)$. The direct observable is the projected surface brightness $\mu$ (in units of erg $\mathrm{cm}^{-2} \mathrm{~s}^{-1}$ ) in an annulus, and it is a function of $\psi$. The luminosity density (in units of $\mathrm{erg}^{-3}$ $\left.\mathrm{s}^{-1}\right) \mathcal{L}(r)=\mathcal{L}\left(\sqrt{\psi^{2}+z^{2}}\right)$ is then related to $\mu(\psi)$ by

$$
\mu(\psi)=2 \int_{0}^{\sqrt{R^{2}-\psi^{2}}} \mathcal{L}\left(\sqrt{\psi^{2}+z^{2}}\right) d z .
$$

If we put this integral in terms of $r$, we obtain the relation

$$
\mu(\psi)=2 \int_{\psi}^{R} r\left(r^{2}-\psi^{2}\right)^{-1 / 2} \mathcal{L}(r) d r=\int_{R}^{\psi} K(r, \psi) \mathcal{L}(r) d r,
$$

where $K(r, \psi)=-2 r\left(r^{2}-\psi^{2}\right)^{-1 / 2}$.

Equation (A2) is a Volterra equation of the first kind, and we solve for $\mathcal{L}(r)$ explicitly for annuli beginning at some radius $R$ and taking $N$ uniform steps of size $h$ inward to $r_{1}=R-h N$. In this case, we selected a radius $R=200 \mathrm{pc} \approx 825^{\prime \prime} .5$ to ensure the entire nebular volume was included. Additionally, we chose a step size of $h=1^{\prime \prime}$ and went inward to $r_{1}=17^{\prime \prime} .5$ (so $N=808$ steps), the radius of the HST PC image.

Figure 16 (top panel) plots the resulting $P_{\mathrm{dir}, 3 \mathrm{D}}$ (and $P_{\mathrm{dir}}$ for comparison) versus the distance $R$ from R136. The bottom panel gives the fractional difference $\left(P_{\mathrm{dir}}-P_{\mathrm{dir}, 3 \mathrm{D}}\right) / P_{\mathrm{dir}}$ for all the points in the top panel. The fractional difference between $P_{\mathrm{dir}, 3 \mathrm{D}}$ and $P_{\text {dir }}$ is small $(\sim 0.1 \%-3.0 \%)$ for regions $\gtrsim 20$ pc from R136, and becomes larger $(\sim 10 \%-60 \%$ for radii $\lesssim 20$ pc. Despite these greater fractional differences at smaller radii, $P_{\text {dir,3D }}$ would still dominate over the other pressure components in Figure 11 at distances $\lesssim 75$ pc from R136. Additionally, the small fractional differences at distances $\gtrsim 20$ pc confirm that our values of $P_{\text {dir }}$ near the shell are accurate.

We note in the above calculation that we necessarily assumed that the luminosity in a shell is spherically symmetric. The surface brightness in 30 Doradus is not actually symmetric though (since stars and star clusters are distributed nonuniformly around the nebula), so our estimated $P_{\text {dir,3D }}$ values are an approximation of the true, deprojected radiation pressure. Nonetheless, the small differences between $P_{\mathrm{dir}, 3 \mathrm{D}}$ and $P_{\text {dir }}$ indicate that uncertainty in the star position along the line of sight does not qualitatively affect our results.

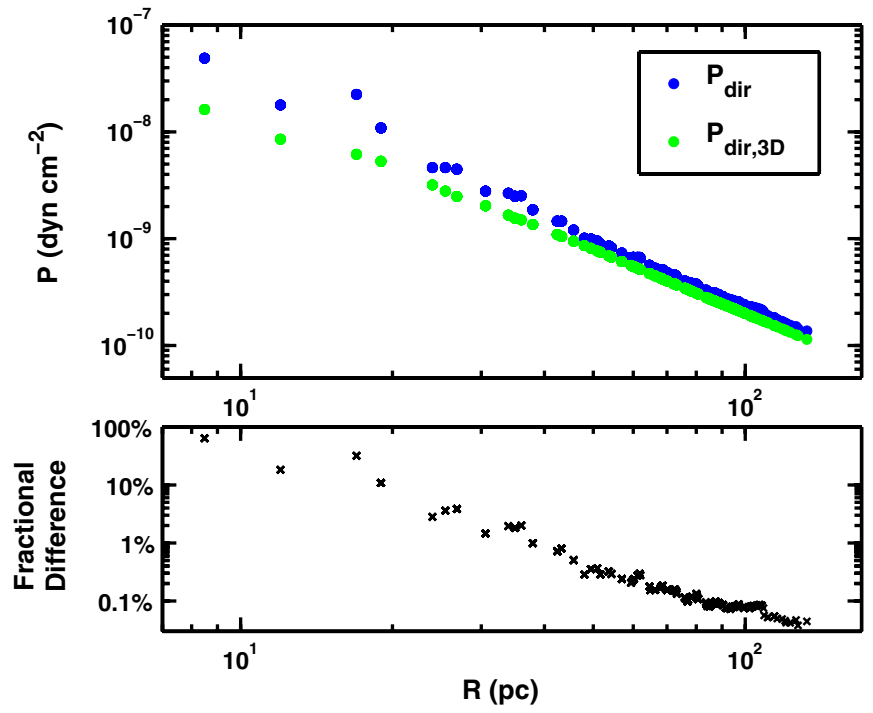

Figure 16. Comparison of $P_{\mathrm{dir}, 3 \mathrm{D}}$ and $P_{\mathrm{dir}}$. Top: the two pressure components vs. distance $R$ from R136. The two have similar radial dependence. Bottom: the fractional difference $\left(P_{\mathrm{dir}}-P_{\mathrm{dir}, 3 \mathrm{D}}\right) / P_{\mathrm{dir}}$ for all the points in the top panel. At radii $\gtrsim 20$ pc from $\mathrm{R} 136$, the fractional difference is small $(0.1 \%-3.0 \%)$, while at radii $\lesssim 20 \mathrm{pc}$, the fractional difference is greater $(10 \%-60 \%)$.

(A color version of this figure is available in the online journal.)

\section{APPENDIX B}

\section{ON THE DEFINITION OF RADIATION PRESSURE}

An alternate definition of radiation pressure than the one used in this paper is to characterize $P_{\text {dir }}$ as the force per unit area exerted by the radiation on matter. This case predicts small $P_{\text {dir }}$ in the H II-region interior, where the density is small, and $P_{\text {dir }}$ only becomes significant near the neutral shell where the radiation is absorbed. To demonstrate this effect, we calculate $f$, the force per unit volume on matter from radiation, as a function of radius in an idealized H II region using Version 08.00 of the photoionization code CLOUDY (Ferland et al. 1998). Assuming photoionization balance, we have

$$
\alpha_{\mathrm{B}} n_{\mathrm{e}} n_{\mathrm{p}}=\frac{S(r)}{4 \pi r^{2}} \sigma_{\mathrm{H}} n_{\mathrm{HI}}
$$

where $\alpha_{\mathrm{B}}=2.6 \times 10^{-13} \mathrm{~cm}^{3} \mathrm{~s}^{-1}$ is the case- $\mathrm{B}$ recombination coefficient of hydrogen at $10^{4} \mathrm{~K} ; n_{\mathrm{e}}, n_{\mathrm{p}}, n_{\mathrm{HI}}$ are the electron, proton, and $\mathrm{H}$ I number densities, respectively, $S$ is the ionizing photon luminosity (in photons $\mathrm{s}^{-1}$ ) passing through a shell at a given radius $r$, and $\sigma_{\mathrm{H}}$ is the $\mathrm{H}$ ionization cross section.

The force density $f$ is given by

$$
f=\frac{\kappa \rho F}{c}=\frac{\kappa_{\mathrm{dust}} \rho F}{c}+\frac{\kappa_{\mathrm{H}} \rho F}{c},
$$

where $\kappa$ is the opacity per unit mass (from dust $\kappa_{\text {dust }}$ or from neutral hydrogen $\kappa_{\mathrm{H}_{\mathrm{I}}}$ ), $\rho$ is the local mass density, and $F$ is the total flux. Given $\kappa_{\text {dust }} \rho=n_{\mathrm{H}} \sigma_{\text {dust }}$ (where $n_{\mathrm{H}}=n_{\mathrm{HI}}+n_{\mathrm{HII}}$ ) and $\kappa_{\mathrm{H}} \rho=n_{\mathrm{HI}} \sigma_{\mathrm{HI}}$

$f=\frac{S(r)\langle h v\rangle}{4 \pi r^{2} c}\left(n_{\mathrm{H}} \sigma_{\text {dust }}+n_{\mathrm{H}_{\mathrm{I}}} \sigma_{\mathrm{HI}}\right)=\frac{\alpha_{\mathrm{B}} n_{\mathrm{e}} n_{\mathrm{p}}\langle h \nu\rangle}{c}\left(1+\frac{n_{\mathrm{H}}}{n_{\mathrm{H}_{\mathrm{I}}}} \frac{\sigma_{\mathrm{dust}}}{\sigma_{\mathrm{H}}}\right)$.

The first term represents the force of ionizing photon absorption by $\mathrm{H}$ atoms and the second term is the force of ionizing photons on the dust. Here, $\langle h v\rangle$ is the mean energy of the ionizing 


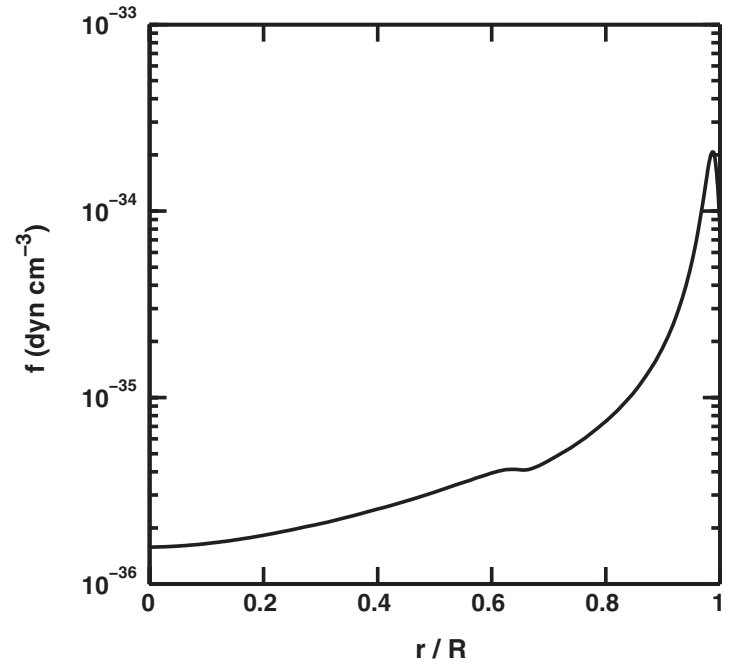

Figure 17. Plot of $f$, the force per unit volume on matter from radiation, vs. fractional radius $r / R$ of the $\mathrm{H}$ II region. The force density $f$ peaks near the $\mathrm{H}$ II shell and is several orders of magnitude less interior to the shell.

photons, assuming that the force in the radiation field is from ionizing photons only. In the following calculation, we set $\langle h v\rangle=15 \mathrm{eV}$, a value typical of an $\mathrm{O}$ star. Similarly, we assume that the force on dust is from ionizing photons, and we adopt a dust cross section at $15 \mathrm{eV}, \sigma_{\text {dust }}=1.0 \times 10^{-21} \mathrm{~cm}^{2} / \mathrm{H}$ (Weingartner \& Draine 2001). In the above expression, we set $\sigma_{\mathrm{HI}}=6.3 \times 10^{-18} \mathrm{~cm}^{-2}$.

The radial dependence of $f$ comes from the density profiles, $n_{\mathrm{e}}(r)$ and $n_{\mathrm{p}}(r)$. To estimate these parameters as a function of radius in CLOUDY, we utilize the OSTAR2002 stellar atmosphere model for a metallicity $Z / Z_{\odot}=0.5$ (Lanz \& Hubeny 2003) and stellar temperature of $T_{*}=35,000 \mathrm{~K}$. In our analysis, we set our idealized H II region to have $S=6.9 \times 10^{49}$ photons s $^{-1}$ and $n_{\mathrm{H}}=10 \mathrm{~cm}^{-3}$. Additionally, we include the effects of interstellar grains in the calculation.

Figure 17 plots the resulting $f$ as a function of fractional radius in the $\mathrm{H}$ II region. The force per unit volume of the radiation increases many orders of magnitude from the center to the edge of the $\mathrm{H}$ II region, with a drastic jump in $f$ at $r / R \gtrsim 0.9$. This plot contrasts Figure 11 , where $P_{\text {dir }}$ falls off as $1 / r^{2}$.

\section{REFERENCES}

Andrews, B. H., \& Thompson, T. A. 2011, ApJ, 727, 97

Ballesteros-Paredes, J., \& Hartmann, L. 2007, RevMexAA, 43, 123

Bradley, T. R., Knapen, J. H., Beckman, J. E., \& Folkes, S. L. 2006, A\&A, 459, L13

Brandl, B. R. 2005, in Astrophysics Space Science Library 329, Starbursts: From 30 Doradus to Lyman Break Galaxies, ed. R. de Grijs \& R. M. González Delgado (Berlin: Springer), 49

Castor, J., McCray, R., \& Weaver, R. 1975, ApJ, 200, L107

Chevalier, R. A., \& Clegg, A. W. 1985, Nature, 317, 44

Chu, Y.-H., \& Kennicutt, R. C., Jr. 1994, ApJ, 425, 720

Cunningham, A. J., Frank, A., Quillen, A. C., \& Blackman, E. G. 2006, ApJ, 653,416

Dale, J. E., Bonnell, I. A., Clarke, C. J., \& Bate, M. R. 2005, MNRAS, 358, 291

Dickel, J. R., McIntyre, V. J., Gruendl, R. A., \& Milne, D. K. 2005, AJ, 129, 790

Draine, B. T. 2010, arXiv:1003.0474

Draine, B. T., \& Li, A. 2007, ApJ, 657, 810

Elmegreen, B. G. 2000, ApJ, 530, 277

Fall, S. M., Krumholz, M. R., \& Matzner, C. D. 2010, ApJ, 710, L142

Fazio, G. G., et al. 2004, ApJS, 154, 10

Ferland, G. J., Korista, K. T., Verner, D. A., Ferguson, J. W., Kingdon, J. B., \& Verner, E. M. 1998, PASP, 110, 761
Freeman, P. E., Kashyap, V., Rosner, R., \& Lamb, D. Q. 2002, ApJS, 138, 185

Gordon, K. D., Engelbracht, C. W., Rieke, G. H., Misselt, K. A., Smith, J.-D. T., \& Kennicutt, R. C., Jr. 2008, ApJ, 682, 336

Guhathakurta, P., \& Draine, B. T. 1989, ApJ, 345, 230

Harper-Clark, E., \& Murray, N. 2009, ApJ, 693, 1696

Hartmann, L., Ballesteros-Paredes, J., \& Bergin, E. A. 2001, ApJ, 562, 852

Haynes, R. F., et al. 1991, A\&A, 252, 475

Helou, G., et al. 2004, ApJS, 154, 253

Hodge, P. W., Balsley, J., Wyder, T. K., \& Skelton, B. P. 1999, PASP, 111, 685

Hodge, P., Kennicutt, R. C., \& Strobel, N. 1994, PASP, 106, 765

Hodge, P., Lee, M. G., \& Kennicutt, R. C., Jr. 1989a, PASP, 101, 32

Hodge, P., Lee, M. G., \& Kennicutt, R. C., Jr. 1989b, PASP, 101, 640

Hodge, P., Strobel, N. V., \& Kennicutt, R. C. 1994, PASP, 106, 309

Hunter, D. A., Shaya, E. J., Holtzman, J. A., Light, R. M., O’Neil, E. J., Jr., \& Lynds, R. 1995, ApJ, 448, 179

Indebetouw, R., et al. 2009, ApJ, 694, 84

Jijina, J., \& Adams, F. C. 1996, ApJ, 462, 874

Johansson, L. E. B., et al. 1998, A\&A, 331, 857

Kennicutt, R. C., Jr. 1984, ApJ, 287, 116

Kennicutt, R. C., Jr., \& Hodge, P. W. 1986, ApJ, 306, 130

Kennicutt, R. C., Jr., Lee, J. C., Funes, S. J., José, G., Sakai, S., \& Akiyama, S. 2008, ApJS, 178, 247

Knapen, J. H., de Jong, R. S., Stedman, S., \& Bramich, D. M. 2003, MNRAS, 344,527

Krabbe, A. C., \& Copetti, M. V. F. 2002, A\&A, 387, 295

Krumholz, M. R., \& Matzner, C. D. 2009, ApJ, 703, 1352

Krumholz, M. R., Matzner, C. D., \& McKee, C. F. 2006, ApJ, 653, 361

Krumholz, M. R., \& Tan, J. C. 2007, ApJ, 654, 304

Kudritzki, R. P., Puls, J., Lennon, D. J., Venn, K. A., Reetz, J., Najarro, F., McCarthy, J. K., \& Herrero, A. 1999, A\&A, 350, 970

Kurt, C. M., \& Dufour, R. J. 1998, RevMexAA Conf. Ser., 7, 202

Lanz, T., \& Hubeny, I. 2003, ApJS, 146, 417

Lazendic, J. S., Dickel, J. R., \& Jones, P. A. 2003, ApJ, 596, 287

Leitherer, C. 1997, RevMexAA Conf. Ser., 6, 114

Leitherer, C., et al. 1999, ApJS, 123, 3

Li, Z.-Y., \& Nakamura, F. 2006, ApJ, 640, L187

Liedahl, D. A., Osterheld, A. L., \& Goldstein, W. H. 1995, ApJ, 438, L115

Malumuth, E. M., \& Heap, S. R. 1994, AJ, 107, 1054

Massey, P., \& Hunter, D. A. 1998, ApJ, 493, 180

Mathis, J. S., Mezger, P. G., \& Panagia, N. 1983, A\&A, 128, 212

Matzner, C. D. 2002, ApJ, 566, 302

Matzner, C. D. 2007, ApJ, 659, 1394

McKee, C. F., \& Williams, J. P. 1997, ApJ, 476, 144

Meixner, M., et al. 2006, AJ, 132, 2268

Meurer, G. R., Heckman, T. M., Lehnert, M. D., Leitherer, C., \& Lowenthal, J. 1997, AJ, 114, 54

Mewe, R., Gronenschild, E. H. B. M., \& van den Oord, G. H. J. 1985, A\&AS, 62, 197

Mewe, R., Lemen, J. R., \& van den Oord, G. H. J. 1986, A\&AS, 65, 511

Motte, F., Andre, P., \& Neri, R. 1998, A\&A, 336, 150

Murray, N., Ménard, B., \& Thompson, T. A. 2010a, arXiv:1005.4419

Murray, N., Quataert, E., \& Thompson, T. A. 2010b, ApJ, 709, 191

Murray, N., \& Rahman, M. 2010, ApJ, 709, 424

Nakamura, F., \& Li, Z.-Y. 2008, ApJ, 687, 354

Onishi, T., Mizuno, A., Kawamura, A., Tachihara, K., \& Fukui, Y. 2002, ApJ, 575,950

Parker, J. W. 1993, AJ, 106, 560

Parker, J. W., \& Garmany, C. D. 1993, AJ, 106, 1471

Peeters, E., Spoon, H. W. W., \& Tielens, A. G. G. M. 2004, ApJ, 613, 986

Quillen, A. C., Thorndike, S. L., Cunningham, A., Frank, A., Gutermuth, R. A., Blackman, E. G., Pipher, J. L., \& Ridge, N. 2005, ApJ, 632, 941

Repolust, T., Puls, J., \& Herrero, A. 2004, A\&A, 415, 349

Rieke, G. H., et al. 2004, ApJS, 154, 25

Rybicki, G. B., \& Lightman, A. P. 1979, Radiative Processes in Astrophysics (New York: Wiley-Interscience), 393

Selman, F. J., \& Melnick, J. 2005, A\&A, 443, 851

Shapley, A. E., Steidel, C. C., Pettini, M., \& Adelberger, K. L. 2003, ApJ, 588, 65

Smith, R. C., and MCELS Team. 1998, PASA, 15, 163

Strobel, N. V., Hodge, P., \& Kennicutt, R. C., Jr. 1990, PASP, 102, 1241

Strobel, N. V., Hodge, P., \& Kennicutt, R. C., Jr. 1991, ApJ, 383, 148

Tan, J. C., Krumholz, M. R., \& McKee, C. F. 2006, ApJ, 641, L121

Testi, L., \& Sargent, A. I. 1998, ApJ, 508, L91

Thompson, T. A., Quataert, E., \& Murray, N. 2005, ApJ, 630, 167 
Townsley, L. K., Broos, P. S., Feigelson, E. D., Brandl, B. R., Chu, Y.-H., Garmire, G. P., \& Pavlov, G. G. 2006a, AJ, 131, 2140

Townsley, L. K., Broos, P. S., Feigelson, E. D., Garmire, G. P., \& Getman, K. V. 2006b, AJ, 131, 2164

Walborn, N. R. 1991, in IAU Symp. 148, The Magellanic Clouds, ed. R. Haynes \& D. Milne (Cambridge: Cambridge Univ. Press), 145

Walborn, N. R., \& Blades, J. C. 1997, ApJS, 112, 457

Wang, Q. D. 1999, ApJ, 510, L139

Wang, P., Li, Z.-Y., Abel, T., \& Nakamura, F. 2010, ApJ, 709, 27
Weaver, R., McCray, R., Castor, J., Shapiro, P., \& Moore, R. 1977, ApJ, 218, 377

Weingartner, J. C., \& Draine, B. T. 2001, ApJS, 134, 263

Whitworth, A. 1979, MNRAS, 186, 59

Williams, J. P., \& McKee, C. F. 1997, ApJ, 476, 1

Wyder, T. K., Hodge, P. W., \& Skelton, B. P. 1997, PASP, 109, 927

Yorke, H. W., Tenorio-Tagle, G., Bodenheimer, P., \& Rozyczka, M. 1989, A\&A, 216, 207

Zuckerman, B., \& Evans, N. J., II. 1974, ApJ, 192, L149 